Review

\title{
A multifaceted review on dihydromyricetin resources, extraction, bioavailability, biotransformation, bioactivities, and food applications with future perspectives to maximize its value
}

\author{
Haolin Zhang ${ }^{1}$, Giovanni Caprioli ${ }^{2}$, Hidayat Hussain ${ }^{3}$, Nguyen Phan Khoi Le ${ }^{4}$, Mohamed A. Farag ${ }^{5,6}$, \\ Jianbo $\mathrm{Xiao}^{7, *}$ \\ ${ }^{1}$ Institute of Chinese Medical Sciences, University of Macau, Macau \\ ${ }^{2}$ School of Pharmacy, University of Camerino, via Sant' Agostino 1, 62032 Camerino, Italy \\ ${ }^{3}$ Leibniz Institute of Plant Biochemistry, Department of Bioorganic Chemistry, Weinberg 3, D-06120 Halle (Saale), Germany \\ ${ }^{4}$ Molecular Preventive Medicine, University Medical Center and Faculty of Medicine, University of Freiburg, 79108 Freiburg, Germany \\ ${ }^{5}$ Pharmacognosy Department, College of Pharmacy, Cairo University, Kasr El-Aini St., Cairo P.B. 11562, Egypt \\ ${ }^{6}$ Department of Chemistry, School of Sciences and Engineering, The American University in Cairo, New Cairo 11835, Egypt \\ ${ }^{7}$ University of Vigo, Nutrition and Bromatology Group, Department of Analytical Chemistry and Food Science, Faculty of Science, E32004 \\ Ourense, Spain
}

\section{ARTICLE INFO}

Article History

Received 1 November 2021

Accepted 1 November 2021

Keywords

Dihydromyricetin

stereoisomerism

pharmacokinetics

bioavailability

biotransformation

pharmacological effects

\begin{abstract}
A B S T R A C T
Natural bioactive compounds present a better alternative to prevent and treat chronic diseases owing to their lower toxicity and abundant resources. (+)-Dihydromyricetin (DMY) is a flavanonol, possessing numerous interesting bioactivities with abundant resources. This review provides a comprehensive overview of the recent advances in DMY natural resources, stereoisomerism, physicochemical properties, extraction, biosynthesis, pharmacokinetics, and biotransformation. Stereoisomerism of DMY should be considered for better indication of its efficacy. Biotechnological approach presents a potential tool for the production of DMY using microbial cell factories. DMY high instability is related to its powerful antioxidant capacity due to pyrogallol moiety in ring $\mathrm{B}$, and whether preparation of other analogues could demonstrate improved properties. DMY demonstrates poor bioavailability based on its low solubility and permeability with several attempts to improve its pharmacokinetics and efficacy. DMY possesses various pharmacological effects, which have been proven by many in vitro and in vivo experiments, while clinical trials are rather scarce, with underlying action mechanisms remaining unclear. Consequently, to maximize the usefulness of DMY in nutraceuticals, improvement in bioavailability, and better understanding of its actions mechanisms and drug interactions ought to be examined in the future along with more clinical evidence.
\end{abstract}

(C) 2021 The Authors. Publishing services by Visagaa Publishing House This is an open access article distributed under the CC BY-NC 4.0 license (https://creativecommons.org/licenses/by/4.0/).

\section{INTRODUCTION}

Rapid urbanization brought on by the modernization process of society has led to the general deterioration of human health manifested by less physical activity, and more high-energy food intake. Consequently, chronic diseases have become one of the most eminent threats that endanger human health to include cardiovascular diseases, cancer, chronic respiratory diseases, diabetes, etc. With regards to the prevention and treatment of chronic diseases, except for changing unhealthy lifestyle, natural bioactive compounds have attracted increasing attention for their low adverse effects and easier daily access [1,2].

Polyphenols belong to secondary metabolites class in planta, which widely exist naturally in dietary sources including fruit and vegetables. Various health-beneficial bioactivities including antioxidation, anti-inflammation, anti-tumor, antimicrobial, and other protective effects, endow polyphenols with great potential to be used in chronic diseases treatment or prevention $[3,4]$. Further, flavonoids represent an important class of polyphenolic secondary metabolites, which can be subdivided into different subgroups depending on the structure. These subgroups are: flavones, flavonols, flavanones, flavanonols, flavanols or catechins, anthocyanins and chalcones [5].

(+)-Dihydromyricetin (DMY) is a flavanonol, a class of flavonoids that possesses multiple bioactivities in humans. It is noteworthy that DMY is used as an abbreviation for (+)-dihydromyricetin in the entire work. In this review, a multi-faced study is presented towards natural sources, stereoisomerism, physicochemical properties, stability, extraction, biosynthesis, pharmacokinetics, biotransformation, pharmacological effects as well as molecular

${ }^{*}$ Corresponding author. Emails: jianboxiao@yahoo.com

Peer review under responsibility of the International Association of Dietetic Nutrition and Safety 
mechanisms, underlying DMY health effects, and application in food industries (Figure 1). Such comprehensive review identifies potential uses for DMY in food and functional food industries with emphasis on future needed research to help maximize its value.

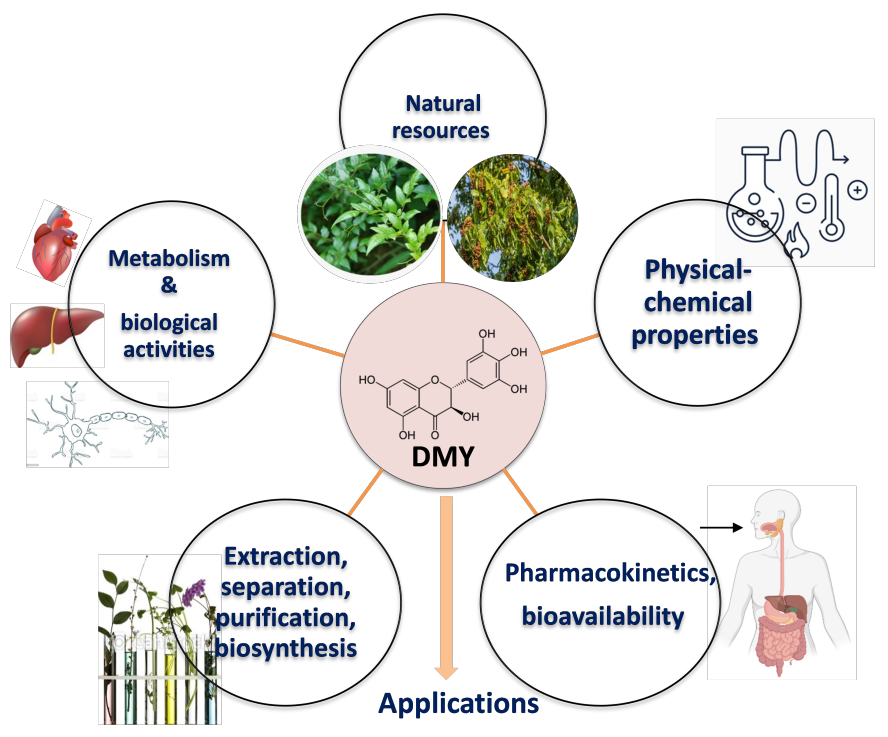

Figure 1 Summary diagram of the review theme and covered topics.

\section{NATURAL RESOURCES OF DMY}

DMY is a natural flavanonol that has been isolated from various plants around the world as follows.

Ampelopsis grossedentata (Hand.-Mazz.) W. T. Wang (A. grossedentata), belonging to the family Vitaceae, is the richest resource of DMY and widely distributed in the south of China. This plant is also named "Tengcha", "vine tea" or "Mao Yan Mei". Moreover, DMY is particularly abundant in its tender stems and leaves, more than $35 \%(\mathrm{w} / \mathrm{w})$ of the dried weight and responsible for the most bioactivities in A. grossedentata [6,7].

Hovenia dulcis (H. dulcis) Thunb, also named as Japanese raisin tree, distributed in China, Japan, Korea, as well as other parts of world, presents another natural resource of DMY [8-10], along with Cedrus deodara (Roxb. ex D.Don) G.Don, Pinaceae, a large evergreen coniferous tree that grows on the slopes of western Himalayas in Eastern Afghanistan, Northern Pakistan, India and southwestern Tibet, China. Furthermore, DMY has been isolated from the latter plant leaves, pine needles, which are often used as drinks in Asia, such as the pine needle tea in China for its beneficial effects [11].

Dietary sources for DMY include vegetables, herbs, as well as fruits, such as berries and grapes [12].

\section{STEREOISOMERISM OF DMY}

Most of previous studies of DMY did not consider stereoisomers type in DMY, with a $(2 \mathrm{R}, 3 \mathrm{R})$-configuration is often considered as a default form of DMY in most articles. In fact, DMY can exist in 4 theoretical stereoisomers $(\mathbf{a}, \mathbf{b}, \mathbf{c}, \mathbf{d})$ [7, 13] of distinct configurations owing to the presence of two chiral centers at C-2 and C-3 as shown in Fig. 2. Previously, few studies have focused on the dextro-isomer / (+) DMY (a), levo-isomer / (-) DMY (b), and their equimolar mixture (racemate) / $( \pm)$ DMY in Fig. 2. Among of them, homochiral (+) DMY with a $(2 \mathrm{R}, 3 \mathrm{R})$-configuration is often considered as the default form of DMY in some articles, showing higher anti-inflammatory activity than $( \pm)$ DMY [14].

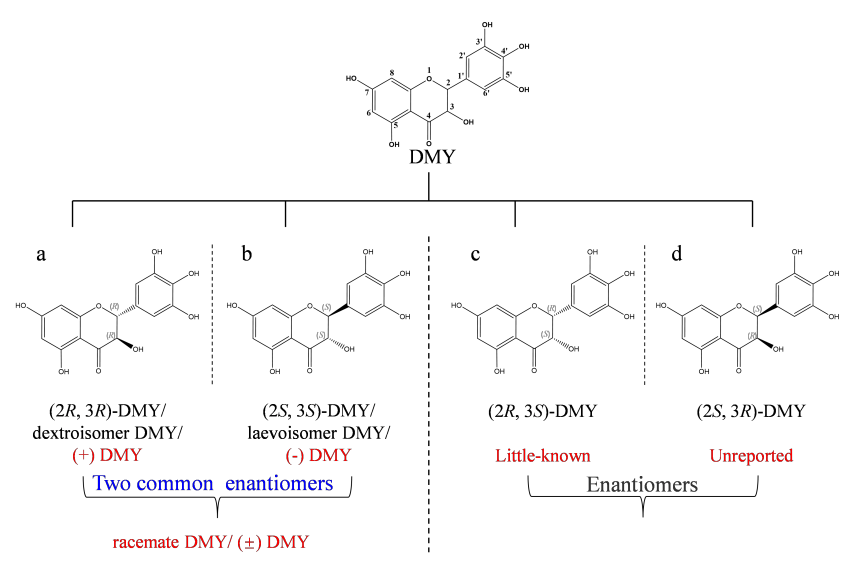

Figure 2 Four theoretical stereoisomers of DMY.

The homochiral enantiomer in pharmacological research is usually produced by extracting the optically pure flavonoid compounds in plants. However, some commercial (+) DMY products actually are racemate $( \pm)$ DMY because the slight changes of metal ions, $\mathrm{pH}$, and temperature during extraction or crystallization process might cause the chiral inversion of DMY [14-16]. Moreover, () DMY enantiopure and racemate mixture of $( \pm)$ DMY were isolated at a ratio of about 1:2 from Ampelopsis grossedentata as identified using HPLC-ESI/MS analysis. Further, (-) DMY exhibited more powerful antimicrobial and antioxidant activities than synthetic antimicrobial (nisin) and antioxidant agents tertbutylhydroquinone. The 2,2-diphenyl-1-picrylhydrazyl (DPPH) scavenging abilities (\%) were observed in the following order: (-) $\mathrm{DMY}>(+) \mathrm{DMY}>( \pm) \mathrm{DMY}>$ tert-butylhydroquinone. Likewise, an antimicrobial assay against $\mathrm{B}$. cereus (AS11846) revealed that (-) DMY was also higher than ( \pm ) DMY. Improved antimicrobial action is likely attributed to that (-) DMY increased the cell permeability and cell leakage, causing cell death [17].

In addition, stereoisomerism of DMY could affect the PK of (+) DMY in rats and the values of $\mathrm{T}_{\max }, \mathrm{AUC}_{0-\infty}, \mathrm{V}_{d}$, were larger than $( \pm)$ DMY groups, which suggested the distinct tissue distribution and elimination due to the stereoisomerism. The results of the PK study also revealed their poor absorptions into the blood, probably high tissue distributions and slow elimination processes [18].

In short, different stereoisomers of DMY appear to exhibit distinct activities and pharmacokinetic characteristics rationalizing for the need to determine the accurate configuration of DMY in bioassays. It is also important that homochiral DMY, especially (+) DMY, must be produced by more ideal enantiopure separation methods for examining in further bioassays or clinical trials. 


\section{PHYSICO-CHEMICAL PROPERTIES AND STABILITY OF DMY}

DMY is a white needle-like crystal obtained from ethanol with very poor water-solubility and stability as well as powerful antioxidative activity [19]. DMY with 2-phenylchromogenone as the core structure belongs to the class IV based on the biopharmaceutical classification system (BCS) due to its low solubility and permeability [7].

\subsection{Solubility of DMY}

The solubility of DMY in solvents obeys the following order: hot water, hot ethanol and acetone (readily soluble) $>$ ethanol, methanol (soluble) > ethyl acetate (very slightly soluble) > chloroform, petroleum ether (insoluble). The solubility of DMY improved with the increase of temperature from $288.15 \mathrm{~K}$ to $323.15 \mathrm{~K}$ and ethanol level in the ethanol/water mixture [20]. The solubility of DMY in buffer solution ( $\mathrm{pH} 3.0,4.0,5.0,6.0)$ at $25^{\circ} \mathrm{C}$ were at $741,725,730$ and $853 \mu \mathrm{g} / \mathrm{mL}$, respectively (Sun et al., 2020a), suggesting that $\mathrm{pH}$ 6.0 showed best solubilization.

\subsection{Antioxidant activity of DMY}

The antioxidant capacity of DMY in vitro has been measured using several in vitro assays showing potential effect though with inconsistency among some as follows. Based on DPPH, 2,2'-azino-bis (3-ethylbenzothiazoline-6-sulfonic acid (ABTS) and ferric reducing antioxidant power (FRAP) methods, the antioxidant capacity of DMY was higher than ascorbic acid and Trolox within the low concentration ranges [21]. The $\mathrm{IC}_{50}$ values of the DMY in DPPH and ABTS assays were at 27.5 and $15.8 \mu \mathrm{M}$, respectively and complexation of DMY with cyclodextrins could enhance the antioxidant effects [22]. In contrast, $\mathrm{IC}_{50}$ values of the DMY in the DPPH and ABTS assays were determined at 7 and $2 \mu \mathrm{M}$, respectively [21]. $\mathrm{EC}_{50}$ values of DMY for scavenging $\mathrm{DPPH}, \mathrm{ABTS}$, $\mathrm{H}_{2} \mathrm{O}_{2}$, and $\mathrm{O}_{2}$ radicals were at $8.18,5.32,7.95$, and $7.79 \mu \mathrm{g} / \mathrm{mL}$, respectively [19]. $\mathrm{IC}_{50}$ of DMY in the DPPH assay was estimated at $3.24 \mu \mathrm{g} / \mathrm{mL}$ and the oxygen radical absorbance capacity (ORAC) value of DMY was close to $4000 \mu \mathrm{moL} / \mathrm{g}$ [23].

Such inconsistency in results may be attributed to slight modifications to the same methods and distinct reaction systems used. Thus, more accessible and universal methods to evaluate the antioxidant capacity of flavonoids in vitro are warranted to assess relative efficacy among DMY isomers.

\subsection{Stability of DMY}

The stability of DMY is known to be notably weaker than most other flavonoids, due to its special structure with six hydroxyl groups, particularly the readily oxidative hydroxyl groups in the pyrogallol moiety in ring B [24]. DMY is highly unstable in solution and to degrade rapidly by decomposition and isomerization of DMY induced by the chiral inversion [16] . Furthermore, other factors affecting the stability of DMY include $\mathrm{pH}$, temperature, metal ions, interaction with macromolecules and other components [7, 25].
Among these factors, $\mathrm{pH}$ plays a critical role in the stability of DMY. DMY is more stable in the acidic $\mathrm{pH}$ range of 1.0-5.0, while readily oxidized and significantly degraded in the alkaline $\mathrm{pH}$ range, especially at 6.0-8.0 [19]. The gastrointestinal (GI) stability study indicated that DMY was stable at $\mathrm{pH} 1.2$, though unstable at $\mathrm{pH}$ 6.8 in a simulated digestive system. Moreover, $\mathrm{pH}$ was suggested to account for the poor stability of DMY in the GI tract and consequently led to its poor bioavailability [26].

DMY is very sensitive to changes in temperature and is rapidly transformed into new products. The stability of DMY in DMSO over 14 days was observed via UPLC-MS/MS to be in the order: $-20{ }^{\circ} \mathrm{C}$ (highly stable) $>4{ }^{\circ} \mathrm{C}$ (relatively stable, a DMY oxidated products $\mathrm{m} / \mathrm{z}=319.04$ slightly increased at positive ion mode $)>24{ }^{\circ} \mathrm{C}(\mathrm{m} / \mathrm{z}=319.04$ significantly increased $)>37{ }^{\circ} \mathrm{C}$ (significantly unstable, several new compounds appeared after 5 $h$ and pronounced changes appeared after 7 and 14 days). The degradation of DMY in phosphate buffer $(\mathrm{pH} 8.0)$ was faster with rising temperature and to disappear quickly within $3 \mathrm{~h}$ [7]. Thus, DMY standard solutions must be stored at $-20{ }^{\circ} \mathrm{C}$ and handed on ice to insure no degradation.

The investigation of common metal ions effect on DMY stability demonstrated that $\mathrm{Ca}^{2+}, \mathrm{Na}^{+}, \mathrm{K}^{+}$and $\mathrm{Mg}^{2+}$ slightly decreased its stability in water while $\mathrm{Fe}^{3+}, \mathrm{Cu}^{2+}$ accelerated degradation of DMY. DMY was almost undetectable in the presence of $\mathrm{Fe}^{3+}$, and only $42 \%$ of DMY was left in the presence of $\mathrm{Cu}^{2+}$ within $1 \mathrm{~h} \mathrm{[7]}$. It was assumed that compared to the mono-hydroxyl group, flavonoids with ortho-hydroxyls in the $\mathrm{B}$ ring were more sensitive to metal ions $\left(\mathrm{Fe}^{3+}, \mathrm{Cu}^{2+}\right)$ which may be due to their chelation with $\mathrm{Fe}^{3+}$ and $\mathrm{Cu}^{2+}$ [27]. A study via ultraviolet (UV) and fluorescence spectra revealed that iron ions could affect the UV spectrum of DMY, suggesting that iron ions could chelate DMY. Interestingly, $\mathrm{pH}$ was found to influence the chelation between DMY and $\mathrm{Fe}^{2+} / \mathrm{Fe}^{3+}$ [28]. Whether difference in stability exists among DMY stereoisomers has yet to be investigated.

Interaction with macromolecules could also play a significant role in improving DMY stability in complex matrix. For example, proteins could form complexes with flavonoids to enhance the stability of flavonoids [29]. Multiple spectroscopic methods, including UV-vis absorption, fluorescence quenching, synchronous fluorescence, Fourier-transform infrared spectroscopy, circular dichroism spectroscopy, as well as molecular docking approaches were used to investigate the interaction (binding constants, sites, forces, distance, etc.) between proteins and flavonoids. Moreover, DMY dramatically quenched human hemoglobin intrinsic fluorescence in a static quenching way by forming the DMY and human hemoglobin complex [30]. Similarly, DMY could also quench the bovine serum albumin fluorescence based on dynamic quenching, static quenching and energy transfer mechanisms. Importantly, hydrophobic forces might have a significant role in the binding process [31]. The fluorescence of human serum albumin was also quenched by DMY due to the formation of the DMY and human serum albumin complex (Chen et al., 2012a). Another study reported that DMY quenched the bovine lactoferrin (BLF) fluorescence to form the BLF-DMY complex based on the typical static quenching mechanism. In addition, the binding affinity of DMY to BLF was found to be weaker than myricetin due to possessing a single bond instead of a double bond between C2 and $\mathrm{C} 3$, which may weak the $\pi-\pi$ conjugation of $\mathrm{B}$ and $\mathrm{C}$ rings and 
reduce the planar proximity of the two rings, leading to a weaker binding ability of DMY to BLF [29].

Apart from factors mentioned above, other complex components, such as amino acids, glucose, growth factors, etc., may affect the stability of DMY in Dulbecco's Modified Eagle Medium (DMEM) by forming a redox system. Previously, polyphenols have been observed to be unstable in cell culture media, which may give unreliable results of the study of their bioactivities [22, 3234]. Likewise, DMY was also found to be rapidly degraded in DMEM supplemented with $10 \%$ fetal bovine serum at $37{ }^{\circ} \mathrm{C}$, forming possible dimers of DMY, oxidation products, ring-cleavage products and isomers.

Therefore, to improve the stability of DMY and avoid the artificial results in cell culture experiments, a few methods including low temperature and adding ascorbic acid were used [7, 25].

\section{EXTRACTION, SEPARATION, AND PURIFICATION}

As a natural flavanonol with diverse health-promoting bioactivities, the efficient extraction, separation and purification of DMY are significant for its industrial production and or laboratory research [13]. DMY was mainly extracted from the plant $A$. grossedentata (especially rich in leaves) and other plants (Fig. 3). Previous investigations showed that leaves collected in April or May contained the highest content of DMY [35-37]. These results need to be confirmed by harvesting leaves from other regions to be more conclusive.

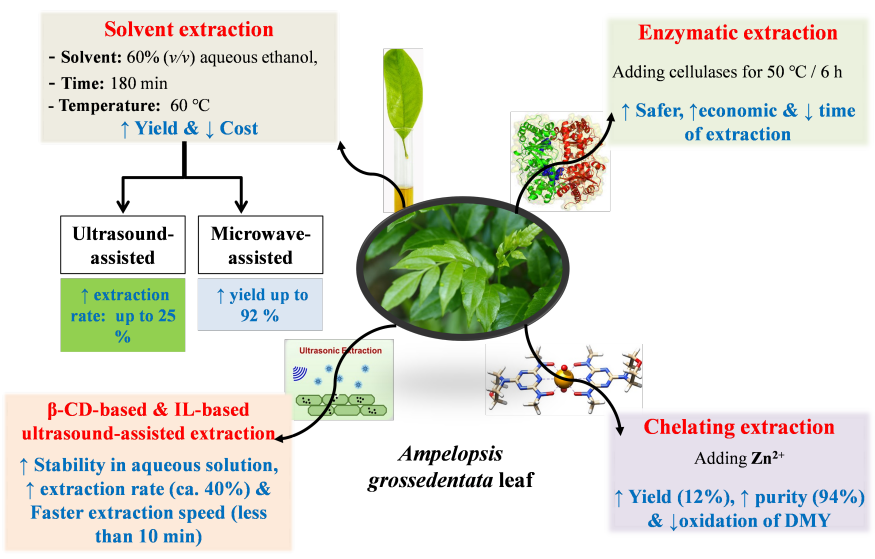

Figure 3 Different methods reported for the extraction of DMY from A.grossedentata leaf.

\subsection{Commonly used techniques}

For extraction, separation and purification of DMY from $A$. grossedentata leaves, several methods including reflux, ultrasound, microwave, supercritical fluid chromatography, high-speed countercurrent chromatography, crystallization and microporous resin adsorption are commonly used alone or in combination. Whereas, for structural identification of DMY, proton nuclear magnetic resonance $\left({ }^{1} \mathrm{H}-\mathrm{NMR}\right)$, single-crystal $\mathrm{X}$-ray diffraction
(XRD), infrared radiation, UV-Vis spectrum and Fourier-transform infrared spectroscopy are used [13, 17, 38]. Identification of exact stereoisomer should be determined using techniques, such as chiral column chromatography and circular dichroism.

\subsection{Solvent based extraction}

A. grossedentata is the plant with the most abundant DMY content. Various solvents have been reported in the extraction of DMY from A. grossedentata, such as hot water, ethanol and other organic solvents including methanol, n-butanol, acetone, and ethyl acetate because DMY is easily soluble in most albeit is slightly soluble in cold water [13]. It was found that extraction using $60 \%$ ethanol in water for $180 \mathrm{~min}$ at $60^{\circ} \mathrm{C}$ from $A$. grossedentata leaves led to maximum DMY recovery rates [39]. For ultrasonic extraction, under the optimum extraction conditions (at $65{ }^{\circ} \mathrm{C}$, extraction of three times, solid-liquid ratio of 1:10 and ultrasound treatment for $45 \mathrm{~min}$ ), the extraction rate of DMY from $A$. grossedentata leaves reached $25.16 \%$ [40]. For dynamic microwaveassisted extraction, under the optimal conditions predicted by response surface methodology $\left(96.8^{\circ} \mathrm{C}, 8.8 \mathrm{~min}\right.$, solid-liquid ratio of $1: 26.4$, and $\mathrm{pH}$ of 5.3 ), the yield was much improved up to $92 \%$ of total DMY in A. grossedentata leaf [41].

Whereas the above organic solvent extraction methods of DMY are popular and relatively simple, they present hazards if left in trace levels in food due to the toxic, inflammable and environmentally unfriendly features of most organic solvents. Thus, it is urge to develop new green methods for DMY extraction from $A$. grossedentata in the food-field application [42].

\subsection{Enzymatic extraction}

The enzymatic extraction is considered both safer and more economical to extract DMY by adding cellulase to hydrolyze dried fruits of A. grossedentata. Compared to the general extraction method (without treatment of cellulase) which needs $24 \mathrm{~h}$ at $100{ }^{\circ} \mathrm{C}$, treatment with cellulase at $50{ }^{\circ} \mathrm{C}$ for $6 \mathrm{~h}$ decreased the temperature and time for hydrolysis of the dried fruit of A. grossedentata and could be directly applied to industrial production [6]. Immobilization of cellulose to reduce its costs and amount needed for extraction should be considered to better optimize such process.

\section{4. $B$-CD-based and IL-based ultrasonic-assisted extraction}

Other green extraction methods of DMY from A. grossedentata approaches are based on two green and helpful components in extraction areas, cyclodextrins and ionic liquids. A few compounds could form inclusion complexes with CDs $(\alpha-\mathrm{CD}, \beta-\mathrm{CD}$ and $\gamma-\mathrm{CD})$ to improve their stability, solubility and bioavailability [43]. DMY could be encapsulated in CDs to form inclusion complex which significantly increase its stability in aqueous solution [44]. Due to its excellent selectivity of extraction, thermostability, solubility, and structural designability, ionic liquids were also widely used in extraction, i.e., extracting flavonoids [45]. Application of $\beta$-CD- 
based and IL-based ultrasonic-assisted methods for extraction of DMY from A. grossedentata both showed higher extraction rate (nearly 40\%) and faster extraction speed (less than 10 min compared to classical solvent extraction methods [42].

\subsection{Chelating extraction}

A new chelating extraction method of DMY is proposed on the basis of the fact that the polyhydroxy structure of DMY makes it much easier to form complexes with metal ions. The metal complexes have been found to improve both stability and antioxidant capacity of original flavonoids and thus to enhance their bioactivities [13, 46]. DMY-Zinc $(\mathrm{Zn})$ complex was a good example. $\mathrm{Zn}-\mathrm{O}$ coordinated bond could be formed between $\mathrm{Zn}^{2+}$ and $3-\mathrm{OH}$ on the $\mathrm{C}$ ring of DMY, which could reduce the delocalization of free radicals on the benzene ring and lead to a lower antioxidant activity of DMY [47]. During the chelating extraction of DMY in the presence of $\mathrm{Zn}^{2+}$, DMY-Zn complex was firstly formed and precipitated. After that, DMY was replaced and released by addition of EDTA-2Na to coordinate with $\mathrm{Zn}^{2+}$. Finally, the chelating extraction method had higher yield (12.2\%) and purity (94.3\%) and less oxidation of DMY than traditional extraction methods [13], posing it to be employed as commercial scale for DMY production.

\subsection{Chiral separation}

Remarkably, as mentioned in section 3 above, identification of the exact isomer type in DMY is warranted and typically performed using chiral separation as highlighted in this subsection. A successful approach to extract homochiral (+) DMY from $A$. grossedentata leaves has been established leading to the acquisition of (+) DMY and ( \pm ) DMY. The (+) DMY and ( \pm ) DMY in solidstate were distinguished using spectroscopic analyses, i.e., ${ }^{1} \mathrm{H}$ NMR and chiral HPLC [16]. Several separation and purification methods reported include high-speed countercurrent chromatography [48, 49], supercritical fluid chromatography [50], recrystallization [17] found successful to yield homochiral DMY.

\subsection{Biotechnology}

In addition to the conventional troublesome extraction methods from plants, employment of biotechnology tools present better option for natural products production as in case of flavonoids. Well-established microbial cell factories can produce flavonoids in well-controlled bioreactors with the advantages of being of high yield, and consistency [13, 51]. Moreover, the subsequent operations after production inside the cells are relatively simple, typically including cell breakage, solvent extraction and separation by chromatography [52]. A transcriptome analysis of A. grossedentata showed that genes encoding main enzymes in phenylpropanoid biosynthetic pathways were also particularly related to the biosynthesis of DMY, which may provide new insight for the metabolic engineering of DMY production in microbial hosts, i.e., bacteria [53]. DMY has been produced using metabolically engineered strains, namely Saccharomyces cerevisiae. $S$. cerevisiae is an excellent host to readily express cytochrome P450 enzymes, which are suitable for production of hydroxylated flavonoids without pronouncedly affecting its growth [54]. The de novo biosynthetic pathway of DMY in $S$. cerevisiae involves two steps: the production of precursor naringenin and the biotransformation from naringenin to DMY. The de novo biosynthesis pathway of DMY was ultimately constructed by the blockage of some branching pathways and the integration of several biosynthesis-associated genes, as shown in Fig. 4. Finally, in a $5 \mathrm{~L}$ bioreactor, a yield of $246.4 \mathrm{mg} / \mathrm{L}$ DMY was achieved with a total flavonoid content at $528.9 \mathrm{mg} / \mathrm{L}$ [54], suggestive that DMY amounted for $50 \%$ of total flavonoids comparable to $35 \%$ in $A$. grossedentata.

\section{PHARMACOKINETICS AND BIOAVAILABILITY}

The pharmacokinetics (PK) studies, including absorption, distribution, metabolism and elimination (ADME), are vital for the bioavailability assessment, design and usage of any drug to ensure its efficacy as the first step. The physiochemical characteristics of DMY play an important role in its PK. Based on the low solubility and low permeability, many PK studies have proven the poor bioavailability of DMY in vivo and to negatively affect its efficacy [18, 55]. The ADME of DMY has been explored likewise in vivo (animal models) and in vitro (simulated gastrointestinal tract and Caco-2 cell model) as detailed in the next subsections.

\subsection{PK studies of DMY in vitro}

The poor absorption of DMY after oral administration may be the main reason of its low bioavailability. DMY absorption is typically mediated via transcellular (membrane diffusion, carrier-mediated, endocytosis) or paracellular mechanisms [56]. Study was mainly performed in the gastrointestinal (GI) tract, particularly the small intestine. This process is under the influences of many factors, such as digestive enzymes, $\mathrm{pH}$, efflux transporters, metal ions, as well as water solubility, lipophilicity and stability of DMY etc. Therefore, simulated GI tract and Caco-2 cell model in vitro results aid to understanding the underlying absorption mechanism of DMY in vivo.

\subsubsection{Simulated digestive system}

Simulated digestive system was used to study DMY stability inside the GI tract. DMY was observed to be highly stable in simulated gastric fluid (SGF, $\mathrm{pH}$ 1.2) while quite unstable in simulated intestinal fluid (SIF, $\mathrm{pH}$ 6.8) for undergoing a pseudo-first-order kinetic degradation. The results suggested that the $\mathrm{pH}$, rather than the presence of pepsin or pancreatin, accounted for the poor stability of DMY in GI tract and DMY being unstable in the duodenum, small intestine, and colon [26]. Consequently, absorption should be targeted in formulation to occur in the stomach to ensure stability of DMY. Besides, degradation of DMY in SIF could be significantly accelerated with the addition of $\mathrm{Fe}^{3+}$ while slowed down supplemented with ascorbic acid in SIF within $6 \mathrm{~h}$ (Sun et al., 2020a). The lipid-water partition coefficient ( $\log \mathrm{P})$, a parameter of drug lipophilicity, could partly reflect the ability of a drug to cross phospholipids membrane and predict its intestinal absorption [57, 


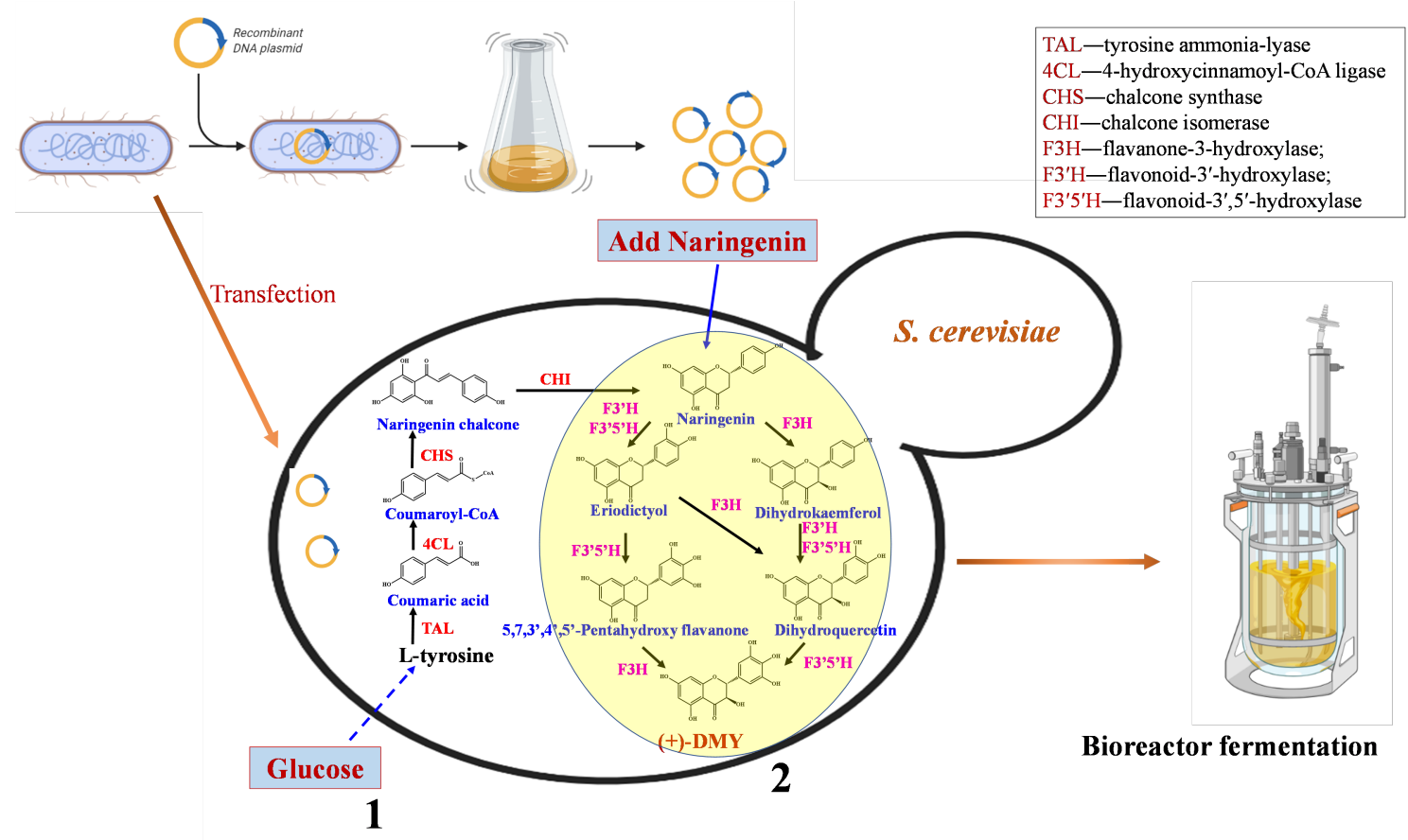

Figure 4 The de novo biosynthesis pathway of DMY in S. cerevisiae. Integrate genes of TAL, 4CL, CHS, CHI, F3H, F3'H, and F3'5'H into the genome of S. cerevisiae. The de novobiosynthesis of DMY: (1) production ofprecursor naringenin from glucose; (2) biotransformation from naringenin to DMY.

58]. The Log P values of DMY in SGF (1.53) and SIF (0.93) (between 0 and 2 but small) suggested that DMY could be partly absorbed but with a poor bioavailability according to the criteria previously described [7].

\subsubsection{Uptake and transport mechanism of DMY across human intestinal Caco- 2 cells}

Caco-2 cell, a human colon carcinoma cell line, could be used to establish a representative transport model system for the small intestinal epithelium [59]. Thus, Caco-2 cell models were applied to investigate and understand the underlying intestinal transport mechanism of DMY, with poor absorption by passive diffusion, being dependent on the time and concentration. The $\mathrm{pH}$ reduction from 8.0 to 6.0 showed no obvious effect on its bidirectional transport $[55,60]$. In addition, efflux transporters could pump drugs out of cells and cause a low oral bioavailability when the drugs are a substrate of efflux transporters. These efflux transporters include P-glycoprotein (P-gp), multidrug resistance protein 1 (MRP1), multidrug resistance protein 2 (MRP2) and breast cancer resistance protein (BCRP) [61]. Flavonoids are often likely to be a substrate of efflux transporters, that further restricting their bioavailability [62]. Thus, the effects of efflux transporters on intestinal absorption of DMY have been evaluated by using their inhibitors to block them, but the results are contradictory. (author?) [55] found that MRP2 and BCRP could hinder the intestinal absorption of DMY, while P-gp and MRP1 did not show obvious effect. Study revealed that P-gp probably decreased the intestinal absorption of DMY based on the fact that after adding a typical P-gp inhibitor verapamil, the absorption of DMY were increased [63], while BCRP and MRP2 were not invovled [60]. In short, efflux transporters are important for the poor intestinal absorption of DMY, but the detailed roles need to be further confirmed towards DMY using in vivo models to be more conclusive as discussed in the next subsection.

\subsection{PK study of DMY in vivo}

To investigate PK of DMY in vivo, animal models, especially rats are commonly used. Meanwhile, LC-MS/MS technique is an efficient way to determine the concentration of DMY and related metabolites in rat plasma, tissues, urine or feces, which is sensitive to detect in such complex matrix and low levels especially if operated in selected ion monitoring mode (SIM), or tandem MS/MS. The results of $\mathrm{PK}$ studies are often expressed as several parameters, including maximum observed concentration $\left(\mathrm{C}_{\max }\right)$, area under the curve (AUC), elimination half-life $\left(t_{1 / 2}\right)$, mean residence time (MRT), the time to $\mathrm{C}_{\max }\left(\mathrm{T}_{\max }\right)$, clearance, and the volume of distribution $\left(\mathrm{V}_{d}\right)$ [64]. In a PK study of DMY, rats in two groups were administrated with DMY by intravenous $(2 \mathrm{mg} / \mathrm{kg}$ ) or oral $(20 \mathrm{mg} / \mathrm{kg})$ way, respectively. Compared to intravenous administration group $\left(\mathrm{C}_{\max }=165.67 \mathrm{ng} / \mathrm{mL}, \mathrm{AUC}_{(0-t)}=410.73\right.$ $\left.\mathrm{ng} \cdot \mathrm{L} / \mathrm{mL}, \mathrm{t}_{1 / 2}=2.05 \mathrm{~h}, \mathrm{MRT}=2.62 \mathrm{~h}\right)$, the oral administration group with a larger dose of DMY (10 times) had dramatically smaller values of $\mathrm{C}_{\max }(21.63 \mathrm{ng} / \mathrm{mL})$ and $\mathrm{AUC}_{(0-t)}(164.97 \mathrm{ng} \cdot \mathrm{L} / \mathrm{mL})$, revealing for the poor absorption of DMY. Moreover, the absolute bioavailability of DMY was calculated to be only $4.02 \%$ which was very poor for typical drugs [65]. The distribution of DMY in different rat tissues, including heart, liver, spleen, lung, kidney, brain, stomach and small intestine as well as in rat urine and feces 
was measured at several time points after the oral administration of DMY $(100 \mathrm{mg} / \mathrm{kg})$. It was found that DMY was rapidly distributed to various tissues, but mostly in the stomach and small intestine. Moreover, 8 metabolites of DMY were detected in urine and feces but almost not detected in plasma. About $90 \%$ of DMY was observed to be eliminated within $12 \mathrm{~h}$ (significantly quickly in the first 2 h), while the unconverted forms of DMY were mainly detected in feces (very little in urine). In addition, DMY was detected in the brain which showed its ability to cross the blood-brain barrier [66], though whether such levels can exert a central nervous system action is not determined.

Moreover, the binding affinities of human hemoglobin and DMY in vivo were markedly related to the distribution, metabolism, and bioactivities of drugs [30] .

\subsection{Gut microbiota affecting PK of DMY}

Gut microbiota might impact the metabolism and biotransformation of DMY though enzyme-catalyzed reactions, thus affecting its bioavailability and efficacy [67]. Compared to the control group, the results of the PK of DMY in pseudo-germ-free rats showed a slight increase of AUC and marked increase of $\mathrm{C}_{\text {max }}$, indicating that gut microbiota might slightly affect the low bioavailability of DMY [68].

\section{APPROACHES TO IMPROVE THE STABILITY \& BIOAVAILABILITY OF DMY}

With such low bioavailability, bioactivities of DMY have been dramatically limited, and its potential for utilization in clinic and food industry is also significantly reduced. Hence, to enhance the bioavailability of DMY, new methods have been already developed, including co-administration of DMY and other substances, as well as novel formulations of DMY to improve its stability, solubility, permeability and bioactivities as detailed in the next subsections (Fig. 5).

\subsection{Novel dosage forms development of DMY}

\subsubsection{Co-crystallization}

Co-crystallization of $( \pm)$ DMY with caffeine or urea dramatically increased the oral bioavailability of $( \pm)$ DMY in rats by supplementation with a proper crystallization inhibitor, polyvinylpyrrolidone K30, to prevent soluble cocrystals from precipitation in aqueous medium (Wang et al., 2016a). Furthermore, the co-crystallization of slightly water-soluble DMY with highly water-soluble pentoxifylline (1:1), DMY-pentoxifylline - $\mathrm{H}_{2} \mathrm{O}$, not only helped to slightly increase the equilibrium solubility of DMY, but also exhibited an in vitro synergistic anti-cancer effect on HepG2 cells at a concentration of $100 \mu \mathrm{M}$ [69].

\subsubsection{Self-nanoemulsifying drug delivery systems}

The self-nanoemulsifying drug delivery system (SNEDDS) of DMY was developed with medium chain triglycerol as oil phase, cremophor-RH40 as surfactant, glycerol as co-surfactant. The DMY-SNEDDS showed better in vitro release, water solubility and stability than DMY [30] .

\subsubsection{Microemulsions}

DMY loaded oil in water based microemulsions (S-DMY) were prepared and showed 3.0-fold permeability coefficients (Papp) than DMY $\left(6.0 \times 10^{-7} \mathrm{~cm} / \mathrm{s}\right)$, indicating higher transport in Caco-2 cell models. In addition, it was demonstrated that S-DMY could reduce the oxidative damage of HepG2 cells which was induced by insulin resistance [70].

\subsubsection{DMY-loaded Soluto/ ${ }^{\circledR} H S 15$ micelles}

The thin-film hydration approach with Solutol HS15 as surfactant was used to form the DMY-loaded Solutol HS15 micelles (DMYMs). The self-assembled DMY-Ms showed more than 25.0-fold water solubility, better sustained release ability containing 5.5fold absorption rate constant and 3.0-fold Papp than that of pure DMY, leading to 2.0-fold relative bioavailability compared with pure DMY. Oral administration of DMY-Ms dramatically alleviated oxidative stress and alcohol-induced tissue damages in mice [71].

\subsubsection{DMY encapsulated zein-caseinate nanoparticles}

The anti-solvent approach was used to form DMY encapsulated zein-caseinate nanoparticles (DZP). DZP significantly improved the diffusion rate of DMY in SGF and SIF and the stability of DMY in SIF. Moreover, DZP exhibited a 1.95-fold increase in the oral bioavailability along with the enhanced DMY metabolites level in plasma [72] .

\subsubsection{Enzymatic acylated DMY}

Enzymatic acylated DMY was efficiently prepared with Aspergillus niger lipase using a cosolvent system of DES (choline chloride: glycerol)-DMSO as the reaction medium. The lipase-catalyzed DMY exhibited higher liposolubility and antioxidant ability [73].

\subsubsection{Gastric floating formulations of DMY}

Owing to the DMY high stability in the acidic environment of the stomach, gastric floating formulations of DMY would allow the selective absorption and sustained release of DMY in the stomach to effectively enhance its bioavailability of DMY and pharmacological activities in vivo. Thus, DMY loaded gastric floating tablets (DMYGFTs) using direct powder compression [12] and gastric floating pills (DMY-GFPs) via extrusion forming and freeze-drying [69] were prepared as sustained release formulations of DMY. Both of DMY-GFTs and DMY-GFPs (better) exhibited significantly longer residence time and better bioavailability than DMY powder in vivo. 


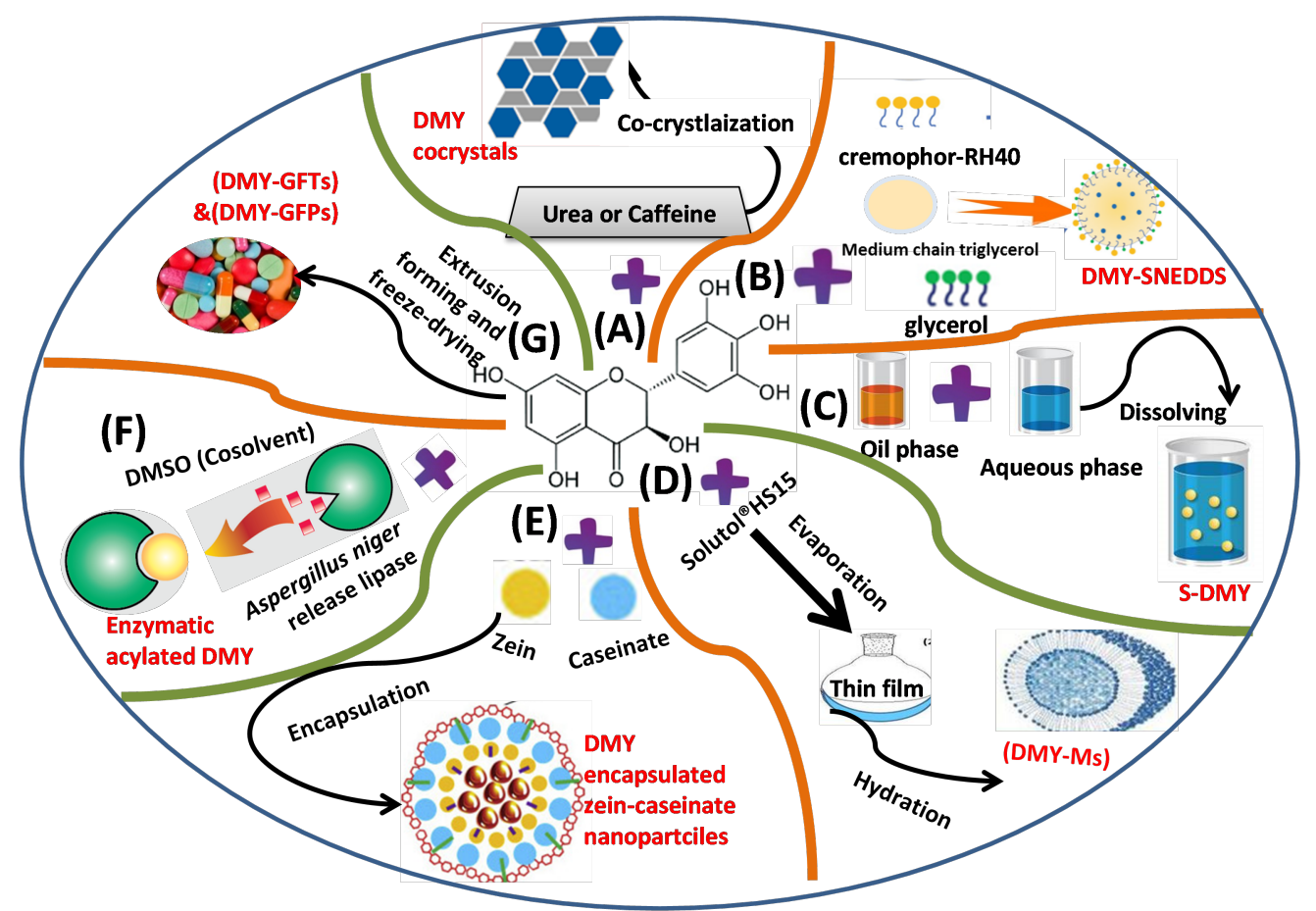

Figure 5 Approaches to improve thestability and bioavailability of DMY.

Furthermore, DMY-GFPs also promoted DMY anti-inflammatory activity in vivo [69].

\subsection{Co- administration of DMY and other compounds}

By co-administration of ascorbic acid and DMY, the bioavailability of DMY was found to be improved in the PK study of rats [7] . The addition of verapamil notably promoted the oral bioavailability of DMY from $3.84 \%$ to $6.84 \%$ in rats. As the inhibitor of $\mathrm{P}$ gp and some CYP enzymes, verapamil was expected to improve the bioavailability through increasing the absorption of DMY by suppressing the efflux effect of P-gp or rat liver metabolism [63].

Therefore, though pharmacokinetics profile and poor bioavailability of DMY highly limited its heath beneficial activities, many novel approaches ought to be considered to improve its application.

\section{BIOTRANSFORMATION OF DMY IN DIFFERENT BIOLOGICAL SYSTEMS}

The biotransformation of drugs in vivo typically consists of phase I, phase II, and phase III reactions, occurring simultaneously or sequentially [74]. The biotransformation primarily occurs in the liver, while other tissues, such as plasma, skin, brain, lung, kidney, GI tract, and gut microbiota also exhibit a critical effect [75, 76]. Metabolites of DMY in vivo which were identified from plasma, urine, feces or gut microbiota are summed up in Fig. 6.

\subsection{Metabolites of DMY in urine, feces and plasma}

After $60 \mathrm{~h}$ of DMY oral administration $(400 \mathrm{mg} / \mathrm{kg})$, urine and feces of rats were collected separately to screen and identify the metabolites of DMY via spectroscopic methods. As shown in Fig. 6, DMY metabolites including 5, 7, 3, 5'-tetrahydroxyflavanonol (4), 5, 7, 4', 5'-tetrahydroxy-3'-methoxyflavanonol (5), 5, 7, 3', 5'-tetrahydroxy-4'-methoxyflavanonol (6) and DMY-5-O- $\beta$-Dglucuronide (9) were identified in urine of rats whereas an isomer of DMY, (2R,3S)-DMY (1), 3, 4, 5, 7, 3', 4', 5'- hepthydroxyflavan (2), 5, 7, 3', 4', 5'-pentahydroxyflavanone (3) were identified in feces of rats. Moreover, metabolites 1, 2, 4 found in feces of rats were also produced by human intestinal bacteria in vitro [64]. In another study, after DMY was orally administered to rats at a dose of $100 \mathrm{mg} / \mathrm{kg}$, the samples of urine, feces, and plasma were collected and the metabolites of DMY were determined based on $\mathrm{MS}^{2}$ data of UPLC-QTOF-MS. In urine and feces, 8 metabolites of DMY were identified and their transformations were predicted to belong to glucuronidation (9), dehydroxylation (3, 4), reduction (2), methylation $(7,8)$, or sulfation $(\mathbf{1 0})$, as well as both dehydroxylation and reduction reactions (11). However, metabolites of DMY were not detected in plasma [66]. Whereas, Sun et al. identified 5 metabolites of DMY in rat plasma by UPLCQTOF-MS, including DMY sulfate (DMY-Sul), DMY glucuronide (DMY-Glu), methylated DMY (DMY-Methyl), methylated DMY glucuronide (DMY-Methyl-Glu) and methylated DMY sulfate (DMY-Methyl-Sul) [72] . We hypothesized that Fan et al. did not detect metabolites of DMY in plasma because of their instability, low content, or improper pretreatment methods. 

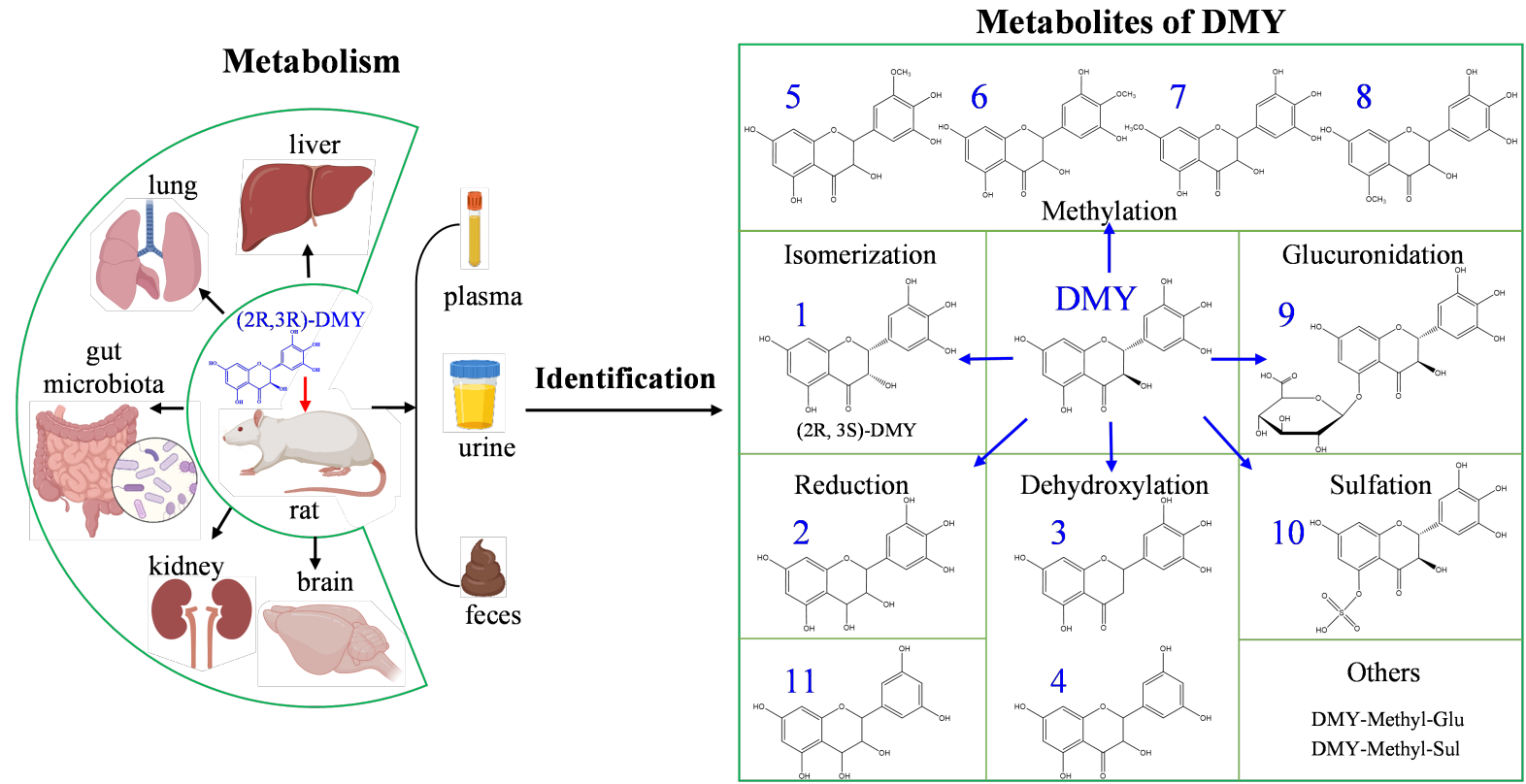

Figure 6 Biotransformation of DMY invivo.
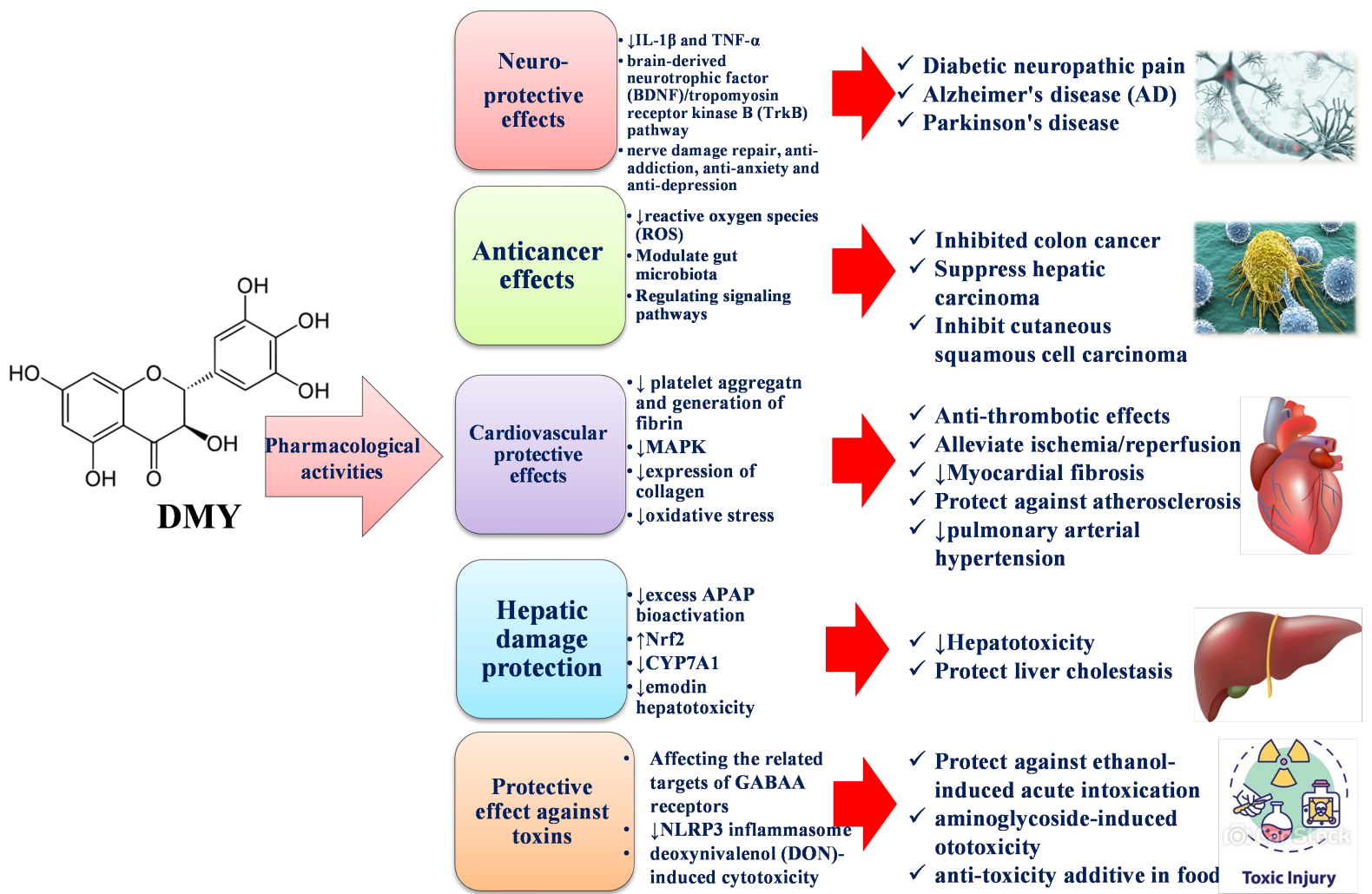

Figure 7 Pharmacological activities andmolecular mechanisms of DMY. 
Table 1 Pharmacological effectsand action mechanisms of DMY

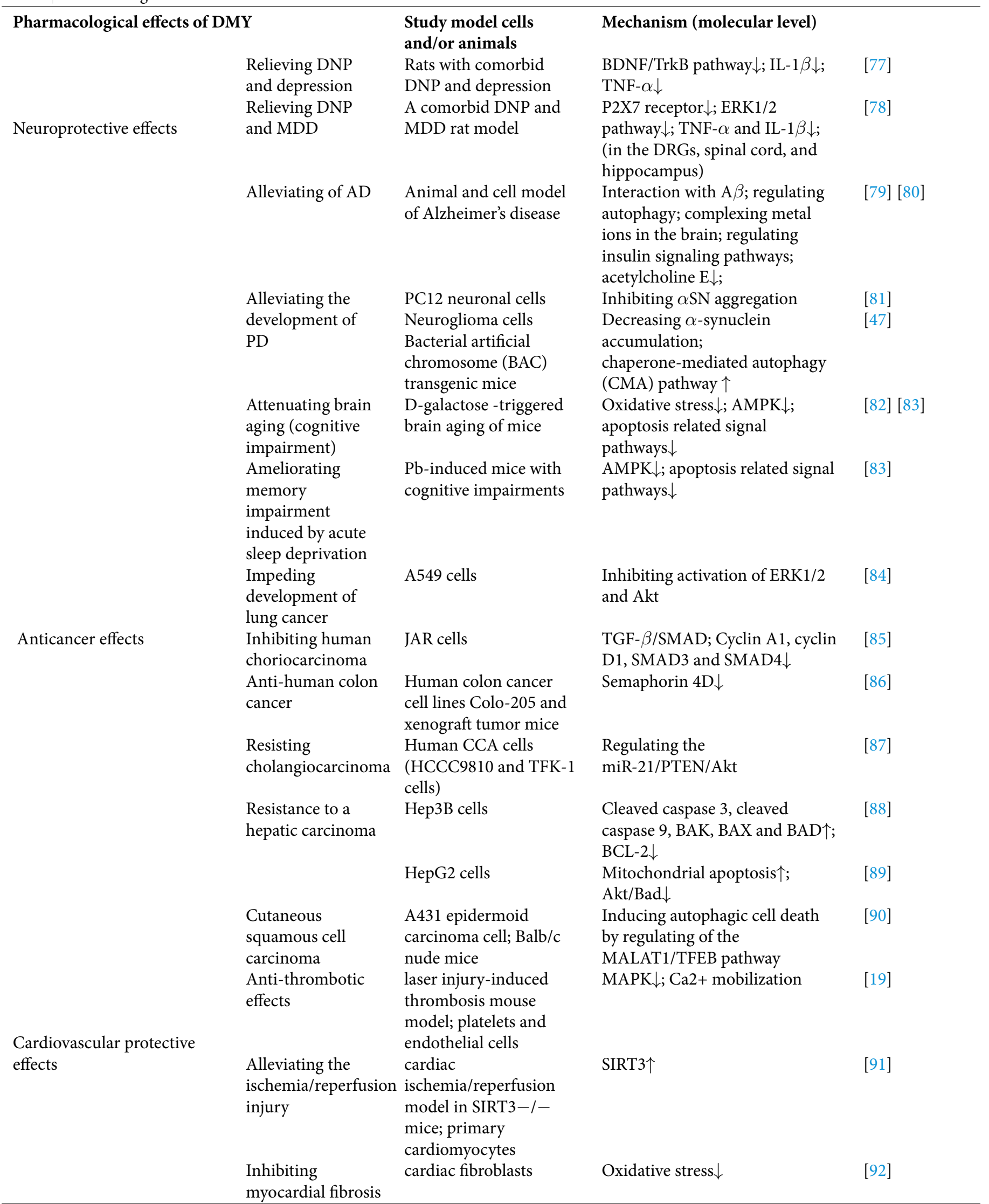


Table 1 continued

Hepatic damage protection

Protective effect of toxic injury

$$
\begin{aligned}
& \text { Inhibiting } \\
& \text { myocardial } \\
& \text { hypertrophy }
\end{aligned}
$$

Alleviating atherosclerosis

Preventing pulmonary arterial hypertension (PAH)

Alleviating cardiotoxicity

Reducing hepatotoxicity

Liver cholestasis Alleviating acute ethanol intoxication Improving ileum injury in chickens

Restraining deoxynivalenolinduced cytotoxicity in IPEC-J2 cells myocardial hypertrophy $\quad$ Oxidative stress $\downarrow$; SIRT3个

[21]

mice induced by

transverse aortic

constriction

HUVECs

$\mathrm{Nrf} 2 / \mathrm{HO}-1 \uparrow$

(ox-LDL-impaired)

HUVECs (sodium

PI3K/Akt/FoxO3a $\uparrow$

nitroprusside-impaired)

monocrotaline -induced

STAT3/MMP9 $\downarrow$

PAH rats; human

pulmonary arterial smooth muscle cells doxorubicin -induced cardiotoxicity; H9C2 cells acetaminophen-induced liver damage mice

L02 cells

Acute ethanol intoxication rats

Chickens with LPS-induced ileum injury IPEC-J2 cells with deoxynivalenol -induced toxicity
SIRT1 $\uparrow$; NLRP3 inflammasome $\uparrow \quad$ [96]

Bax, cytochrome $\mathrm{c} \downarrow$; Bcl-2个; p53 $\downarrow$; CDK4, CCND1 and PCNA $\uparrow ; \operatorname{PPAR} \alpha$ and $\operatorname{PPAR} \gamma \uparrow$; SREBP-1c $\downarrow$; acetaminophen bioactivation $\downarrow$ Nrf2个; CYP7A1 $\downarrow$ GABAARs $\uparrow$

NLRP3 inflammasome $\downarrow$; TLR4/NF- $\kappa \mathrm{B} \uparrow$

Stabilizing metabolism of glutamate, arachidonic and histidine 


\subsection{Metabolites of DMY in fecal matter}

The interaction of dietary polyphenols and gut microbiota has attracted many attentions in recent years. Previous studies have found that polyphenols affected the gut microbial composition and the gut microbiota also influenced the biotransformation of polyphenols [102]. In another study, incubation of DMY and rat fecal microflora in vitro led to the generation of 3 metabolites of DMY based on reduction and dehydroxylation $(2,3,11$ in Fig. 6), and the latter was the dominant form [68].

\section{PHARMACOLOGICAL EFFECTS \& ACTIONS MECHANISMS OF DMY}

DMY is a well-known flavanonol from plants with various beneficial effects, and few side effects. Major effects include antioxidative, antiinflammatory, organ protective (particularly liver), neuroprotective, injury-repairing, anticancer, antimicrobial, detoxifying, cell deathmediating, as well as lipid and glucose metabolism-regulatory activities [14, 95], as shown in Fig. 7.

\subsection{Neuroprotective effects}

The nervous system includes the central nervous system and the peripheral nervous system. DMY has a variety of pharmacological effects on the central nervous system, such as nerve repair, antiaddiction, anti-anxiety and anti-depression.

Diabetic neuropathic pain (DNP) and depression are two common complications of diabetes. After treatment with DMY, the symptoms of DNP and depression comorbid rats were improved. These effects resulted from the reduction level of the proinflammatory cytokines (i.e., IL-1 $\beta$ and TNF- $\alpha$ ) and the inhibition of brain-derived neurotrophic factor (BDNF)/tropomyosin receptor kinase B (TrkB) pathway in the nervous system [77]. Moreover, DMY was demonstrated to alleviate comorbid DNP and major depressive disorder (MDD) symptoms in rats by inhibition of $\mathrm{P}_{2} \mathrm{X}_{7}$ receptor expression, suppression of ERK1/2 signaling pathway, reduction of TNF- $\alpha$ and IL- $1 \beta$ in the dorsal root ganglia, spinal cord, and hippocampus [78]. Another study on MDD demonstrated that DMY exerted an antidepressant effect quicker than a positive drug, namely venlafaxine. This effect was due to the increased expression level of BDNF probably though activating the extracellular signal-regulated protein kinase (ERK1)/2-CREB pathway, suppressing glycogen synthase kinase-3 (GSK-3 $\beta$ ), and then inhibiting neuroinflammation [103].

Alzheimer's disease (AD) is the cause of $60-70 \%$ of cases of dementia. DMY improved the symptoms of AD in animal model of Alzheimer's disease via the up-regulation of AMP-activated protein kinase (AMPK)/sirtuin (SIRT)1 pathway [79]. The possible mechanisms of DMY attenuating $\mathrm{AD}$ mainly include inhibiting the formation of $\mathrm{A} \beta$ oligomers, anti-inflammation, antioxidation, affecting cell autophagy, complexing metal ions in the brain, regulating insulin signaling pathways, regulating the balance of intestinal flora, inhibiting the activity of acetylcholine E, and suppressing the acceleration of bacteria and viruses on $\mathrm{AD}$ [80].
Parkinson's disease (PD) is a progressive nervous system disorder that influences movement. DMY could exhibit inhibitory effects on PD through different mechanisms [104]. For example, DMY was found to inhibit the $\alpha$-synuclein fibrillogenesis, which is related to PD development. Moreover, DMY dramatically reduced the $\alpha$-synuclein -induced cytotoxicity of PC12 neuronal cells [81]. Another study also reported DMY inhibitory effects on $\alpha$-synuclein accumulation and aggregation as well as activation of CMA pathways in neuroglioma cells and bacterial artificial chromosome (BAC) transgenic mouse brain (Wu et al., 2019). Therefore, these results suggested the potential of DMY for alleviating the development of PD.

Oxidative stress and chronic neuroinflammation are significant factors for promoting aging brain. Interestingly, DMY (at low doses of $42 \mathrm{mg} / \mathrm{kg}$ ) pronouncedly improved the cognition impairment of aging brain in $\mathrm{D}$-galactose -induced mice and reduced the damage of hippocampal neurons probably by modulating oxidative stress and neuroinflammation in hippocampus [82]. In addition, DMY could alleviate lead $(\mathrm{Pb})$-induced behavioral disorders in mice by ameliorating oxidative damage, inhibiting inflammation (partly through the AMPK pathway) and apoptosis-related signaling pathways [60].

\subsection{Anticancer effects}

DMY exhibits broad anticancer activities in multiple cancer cells and animal models of cancer. With excellent antioxidant and anti-inflammatory activities, DMY played a significant role in tumor chemoprevention by eliminating reactive oxygen species, influencing gut microbiota, and regulating signaling pathways in cells [19].

Though the original intention of fibroblasts is to help recover the tissue injury, the improper activation of fibroblasts in tumor tissue may indirectly promote cancer development $[105,106]$. DMY was thought to exert a therapeutic potential against lung cancer for its capacity to impede fibroblasts proliferation in A549 lung cancer cells via inhibiting the activation of ERK1/2 and Akt. [66].

DMY exerted inhibitory effects on human choriocarcinoma JAR cells proliferation based on the induction of cell cycle arrest. The mechanism was possibly associated with the transforming growth factor beta (TGF- $\beta$ )/ mothers against decapentaplegic (SMAD) signaling pathway by reducing the expression of cyclin A1, cyclin D1, SMAD3, and SMAD4 [85], though more studies are needed in grafted animal cancer models to be conclusive.

DMY inhibited colon cancer in both in vitro Colo-205 colon cancer cells and in vivo Colo-205 cells xenografted mice model by alleviating the oxidative stress and inflammation, likely mediated via downregulating the expression of Semaphorin $4 \mathrm{D}$ [86]. Furthermore, DMY had a synergistic action with CPT-11 (irinotecan), a typical drug for colorectal cancer, in two kinds of colorectal cancer mouse models of azoxymethane and dextran sodium sulfate chemically induced cancer and adenomatous polyposis coli (APC) multiple intestinal neoplasia (Min)/+ spontaneous cancer [107].

DMY showed resistance to hepatic carcinoma by suppressing cell proliferation, migration, invasion, and stimulating cell apoptosis 
in human hepatoma cells. In two human cholangiocarcinoma HCCC9810 and TFK-1 cells, the mechanism of anti-cancer actions of DMY was through the regulation of microRNA-21(miR-21)/ phosphatase and tensin homolog (PTEN)/Akt. While in Hep3B cells, it was via modulating the apoptotic signaling pathways of cell apoptosis and metastasis $[87,88]$. Besides, apoptosis induction in human hepatocarcinoma HepG2 cells by DMY through activating the mitochondrial apoptosis and suppressing the Akt/Bad signaling pathway [89].

With regards to cutaneous squamous cell carcinoma, DMY promoted cell death in the A431 epidermoid carcinoma cell model through excessive autophagy caused by regulating the metastasis associated lung adenocarcinoma transcript 1 (MALAT1)/ transcription factor EB (TFEB) pathway [90].

Remarkably, DMY appears as a promising adjuvant agent in cancer management in clinic for its capacity to reverse multidrug resistance $[108,109]$, that warrants for further examination.

\subsection{Cardiovascular protective effects}

DMY possesses anti-thrombotic effects due to its ability of endothelial protection. Compared to typical antithrombotic and antiplatelet drugs, DMY hindered thrombus formation by preventing aggregation of platelet and generation of fibrin in the mouse model of laser-induced thrombosis. For the mechanism of action, DMY suppressed mitogen-activated protein kinase (MAPK) and attenuated $\mathrm{Ca}^{2+}$ increase in platelets and endothelial cells [19].

A study based on the cardiac ischemia/reperfusion model in SIRT3-/-mice and in primary cardiomyocytes revealed that by promoting SIRT3, DMY improved mitochondrial function and inhibited oxidative stress via the promotion of SIRT3, consequently resulted in the alleviation of ischemia/reperfusion injury [91]. DMY exhibits an inhibitory effect on myocardial fibrosis as manifested by decrease in the expression of collagen and to suppress proliferation in angiotensin II-induced cardiac fibroblasts through the reduction of oxidative stress [92]. DMY is a potential therapy for myocardial hypertrophy, with improving the transverse aortic constriction-induced myocardial hypertrophy in the mice model, which was associated with oxidative stress reduction and SIRT3 pathway elevation [21]. DMY has the potential to protect against atherosclerosis attributed to vascular endothelial cells oxidative stress injuries. In a previous study, DMY decreased oxLDL-induced human umbilical vein endothelial cells (HUVECs) damage by activating Akt and ERK1/2, which subsequently stimulated the Nrf2/HO-1 pathway [93]. Meanwhile, another study demonstrated that DMY reduced sodium nitroprussideinduced HUVECs oxidative injury by acting as a stimulator of the $\mathrm{PI} 3 \mathrm{~K} / \mathrm{Akt} /$ FoxO3a signaling pathway [94].

DMY may function as a preventive agent for pulmonary arterial hypertension (PAH). Li et al. suggested that the pretreatment of DMY inhibited monocrotaline-induced PAH in rats and IL6-mediated migration of primary human pulmonary arterial smooth muscle cells via the down-regulation of STAT3/MMP9 signaling pathway [95]. DMY could probably be used as an alleviator for severe cardiotoxic side effects which may lead to cardiomyopathy when using doxorubicin for chemotherapy. DMY not only dramatically protected structure and functioned of rat myocardium against doxorubicin -impaired cardiotoxicity, but also inhibited the toxicity in myocardial doxorubicin-treated H9C2 cells through the suppression of NLRP3 inflammasome-mediated inflammation via upregulating the SIRT1 pathway [96].

\subsection{Hepatoprotection}

DMY has a protective effect on hepatotoxicity. For example, a study found that DMY prevented mice from acetaminopheninduced liver damage via multi-target and multi-pathway actions. For the mechanism of action, DMY partly inhibited the excess acetaminophen bioactivation by regulating related enzymatic activities, downregulating the hepatocyte necrosis signaling pathways, increasing p53 related regeneration pathways, and improving the imbalance of lipid homeostatic metabolism via suppressing sterol regulatory element-binding protein-1c (SREBP1c), and enhancing peroxisome proliferator-activated receptor (PPAR) $\alpha / \gamma$ expression [97].

In addition, DMY exhibited a potential protection on liver cholestasis. Emodin is a hepatotoxic compound which possibly stimulates bile acid production leading to cholestasis. DMY has been showed a protective effect from the emodin-induced hepatoxicity on the normal human hepatic LO2 cells via the upregulation of Nrf2 and downregulation of CYP7A1 [98]. Besides, metabolomic results of another study revealed that DMY could also reversed the damage of emodin-induced liver impairment in rats due to the regulation of 18 proteins as well as the metabolism of glutathione, pyrimidine, and tryptophan [110].

\subsection{Protective effect of toxic injuries}

DMY may be a new therapeutic approach with fewer side effects for acute ethanol intoxication. It was demonstrated that DMY could protect rats against ethanol-induced acute intoxication by affecting the related targets of $\gamma$-Aminobutyric acid type A (GABAA) receptors (GABAARs) [111]. Furthermore, an investigation on the biotransformation of DMY related to the acute ethanol antiintoxication in mice demonstrated that 3 metabolites of DMY influenced the increased expression of GABAARs [99].

DMY was also demonstrated to function as a preventive and therapeutic agent of hearing impairment caused by aminoglycosideinduced ototoxicity [112]. DMY protected chickens from the ileum injury induced by Escherichia coli lipopolysaccharide (LPS), via the suppression of NLRP3 inflammasome and activation of TLR4/NF$\kappa \mathrm{B}$ signal axis [100]. DMY had a practical value as an anti-toxicity additive in food or feed, because it could reduce the deoxynivalenolinduced cytotoxicity in porcine jejunum epithelial cell line, IPEC-J2 possibly via limiting the metabolism of glutamate, arachidonic, as well as histidine [101].

\subsection{Miscellaneous}

Asides from the major effects described above, DMY appears promising for the treatment of inflammatory diseases, such as asthma [109], osteoarthritis [45], rheumatoid arthritis [113], 
acute pancreatitis [114], and mastitis [115]. Other disorders in which DMY appeared functional included human melanoma [116] and hypertrophic scar [117]. DMY also exhibited anti-viral and anti-bacterial activities, including anti-influenza A virus [118], anti-spoilage bacteria (mainly P. antarctica) [119] and antiStaphylococcus aureus [120].

DMY is a promising anti-obesity agent [121] and therapeutic mean for type 2 diabetes $[60,122]$ based on its ability to regulate lipid and glucose metabolism. Furthermore, results of a randomized controlled trial carried out in patients with nonalcoholic fatty liver demonstrated that DMY supplementation ameliorated the insulin resistance, glucose, lipid metabolism and liver function, as well as restrained hepatic steatosis [123].

Particularly, the interaction of DMY and gut microbiota should not be ignored, because DMY could significantly affect the composition, abundance, and diversity of gut microbiota [68]. Dong et al. have demonstrated that DMY could alter the abnormal gut microbiota and bile acids metabolism to improve intestinal barrier function and colonic inflammation in dextran sulfate sodium (DSS)-induced colitis mice (Dong et al., 2021). Gut microbiota also plays a role in DMY metabolism, thus, there appears to be a two-way relationship between metabolism of DMY and gut microbiota.

Interestingly, a recent study revealed that DMY had an anti-aging effect on a Drosophila model, which might have similar outcomes in mammals and humans [124], that has yet to be tested.

\section{TOXICOLOGY AND SAFETY}

Considering that DMY is a rich component in the commonly consumed healthy "Tengcha" tea_A. grossedentata, its toxicity and safety must be clearly investigated. Previously, acute oral toxicity tests, long-term toxicity tests, and genotoxicity tests have been carried out on mice or rats to evaluate DMY safety level.

Acute oral toxicity tests of DMY suggested for its safety in mice and rats. In an acute oral toxicity test, 20 mice were orally given 21.5 $\mathrm{g} / \mathrm{kg}$ of body weight (bw) water extract of A. grossedentata for 14 days. There were no symptoms of poisoning or death occurred in mice, indicating that the extract was non-toxic and the acute oral $\mathrm{LD}_{50}$ of extract was larger than $21.5 \mathrm{~g} / \mathrm{kg} \cdot \mathrm{bw}$ [125]. Another study investigated the acute oral toxicity of DMY on 20 rats. After orally given $5.0 \mathrm{~g} / \mathrm{kg}$.bw DMY for 14 days, all rats were normal [126].

A few long-term toxicity tests also demonstrated the safety of DMY in vivo. In a study, total flavonoids of A. grossedentata were administered to mice at a dosage of $1.5 \mathrm{~g} / \mathrm{kg}$ (high) or 0.3 $\mathrm{g} / \mathrm{kg}$ (low) for 12 weeks. No significant difference was found in index of DMY treated groups compared to the untreated group, suggestive that DMY is nontoxic [127]. In another experiment, no obvious side effects were observed in Wistar rats after treated with A. grossedentata for 90 days [125]. Moreover, genotoxicity tests including mutagenicity tests (Ames test), micronucleus test, and sperm malformation test, also showed negative results in $A$. grossedentata-treated rats [125].

\section{DMY APPLICATIONS IN FOOD INDUSTRIES}

Due to the antibacterial effect, antioxidant effect, safety of DMY, it has a broad potential in food industries as outlined in the next subsections and summarized in Fig. 8.

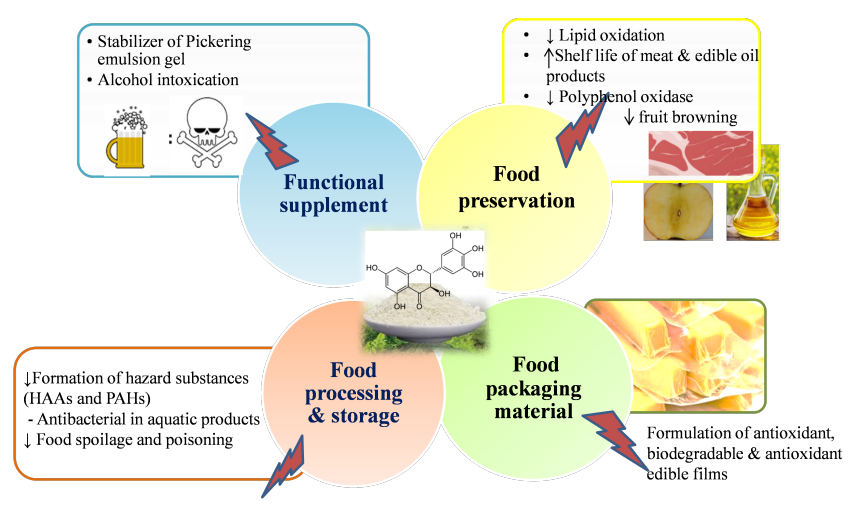

Figure 8 DMY applications in foodindustries.

\subsection{Fresh-keeping during food storage and processing}

The strong antioxidant capacity of DMY makes it attractive for food preservation, including meat, edible oils, and plant food.

\subsubsection{Inhibition of spoilage in meat products}

During the storage of a processed product, abundant nutrients in food, especially proteins and lipids in meat products are readily oxidized, finally leading to spoilage [128]. To solve this problem, the usage of natural antioxidants in meat and meat products is considered as a good choice. The strong antioxidant activity of DMY endowed itself with a capacity to attenuate the oxidation of lipid and protein, in addition to improved sensory quality of meat and prolongation of meat products shelf life [129].

\subsubsection{Inhibition of lipid peroxidation in edible oils}

With the addition of DMY, the degree of lipid oxidation of canola and sunflower oils both dramatically reduced on day 32 and 24, respectively, in comparison to untreated oil. These results indicated the excellent potentiality of DMY to prevent the lipid peroxidation of edible oils [130].

\subsubsection{Anti-browning agent in vegetables and fruit}

In the processing and storage of fruit and vegetables, browning is often an intractable problem that negatively affects their sensory properties. While enzymatic browning is the main way of the browning of fruit and vegetables because of the action of the 
polyphenol oxidase (PPO) presenting in these products. According to this, the PPO activity must be inhibited to develop an antibrowning method. Thus, the utilization of natural plant extracts as PPO inhibitors has been considered as an effective way for that [131, 132]

Comparing the effect of DMY and/or ascorbic acid on the browning of apple slices at $25^{\circ} \mathrm{C}$ for $24 \mathrm{~h}, \mathrm{DMY}$ at a lower concentration of $0.05 \%$ revealed a significantly better anti-browning capacity than ascorbic acid (0.5\%). Moreover, the anti-browning effect was even stronger when DMY and ascorbic acid were used in combination, suggestive that DMY could be considered as an anti-browning agent in plant food [11].

\subsection{Development of green and active packing for food preservation}

To prepare eco-friendlier packaging for food preservation, biodegradable materials including edible films and coatings with additional beneficial functions are encouraged [133, 134]. Studies reported that the addition of DMY into the formulation of composite films consisting of konjac glucomannan and gellan gum not only enhanced the thermostability and water resistance, but also improved antioxidant and antibacterial activities of the films [135]. Similarly, gelatin-based active edible films which were prepared by DMY-load Pickering emulsions stabilized by dialdehyde cellulose nanocrystals exhibited strong antioxidant ability in a DMY dose-dependent way [136].

\subsection{Antibacterial agent in food industries}

With a marked antibacterial effect, DMY could be used as an antibacterial agent in food processing and storage. For instance, DMY could suppress the spoilage bacteria, mainly Pseudomonas antarctica in aquatic products by destroying its biofilm structure and regulating the pathways of respiratory metabolism of biofilm cells. This provides a new idea for aquatic products preservation [119]. With analogous mechanism, DMY revealed powerful antibacterial and bactericidal properties against a usual pathogenic Staphylococcus aureus which causes food poisoning because of its toxin production. Furthermore, DMY also suppressed the adhesion of S. aureus on the surface of stainless steel [120]. These evidences indicated the feasibility of DMY as an antibacterial agent in food processing and storage.

\subsection{Antioxidation and hazardous substance reduction in food thermal processing}

In food thermal processing, multiple hazardous substances with potential carcinogenicity or mutagenicity can be generated, including heterocyclic aromatic amines (HAAs), acrylamide, advanced glycation end products (AGEs), and polycyclic aromatic hydrocarbons (PAHs), etc. [137]. DMY has been reported to inhibit the formation of these harmful substances, for example, formation of several types of genotoxic HAAs in fried beef patties [138]. Likewise, the fortification of DMY in cookies resulted in a pronounced reduction in lipid and protein oxidation as well as AGEs production [139]. Furthermore, the addition of vine tea extract significantly enhanced the sensory features of bread. Meanwhile, the low dose of vine tea extract (1.25 g/kg flour) reduced harmful acrylamide by $58.23 \%$ [140].

\subsection{Stabilizer in preparation of food-grade Pickering emulsion gels}

Pickering emulsion gels is a novel type of emulsion gels that is economical, stable, environment-friendly, insensitive, and using solid particles as stabilizer [141]. Pickering emulsions stabilized by plant-based particles have great potential for use as solid animal fat substitutes and carriers of nutraceutical substances in food industries. The available plant-based particles include common proteins and polysaccharides as well as less used polyphenols [142].

A study revealed that using DMY as a stabilizer of Pickering emulsion gel appears promising for the production possessing as attributed to protective carriers and additional healthy beneficial functions from DMY [141]. Furthermore, DMY notably enhanced the gel structure of starch-based Pickering emulsion gels by constructing DMY and high-amylose corn starch composite particles as a stabilizer [141].

\subsection{Functional supplement for alleviating alcohol intoxication}

Plant sources of DMY have been used as functional supplements, for instance, alleviators for alcohol intoxication in East Asia. In addition, DMY has been applied as a legal alcohol consumption support supplement available in market to relieve alcoholic hangover in the USA. However, the usage of extract of H. dulcis, a plant rich in DMY, was considered but not approved so far by the European Union because it lacked basic PK study and integrated clinical safety assessment [102] [10].

\section{CONCLUSION AND FUTURE PERSPECTIVES}

DMY is mainly isolated from $A$. grossedentata for its high content though warranting for search of other related genotypes for sources of this valuable drug in the future. Stereoisomerism of DMY should be concerned for more accurate results and interpretation of biological effects especially in crude plant extract.

A specific structure with six hydroxyl groups, particularly pyrogallol moiety in ring B, endows DMY with high instability and powerful antioxidant capacity. Among many environmental factors, $\mathrm{pH}$ has the most significant effect on the stability of DMY. The low solubility and low permeability lead to poor bioavailability of DMY in vivo. Hence, many approaches have been developed to enhance its bioavailability.

Metabolism of DMY appears to mainly involve isomerization, reduction, dehydroxylation, methylation, glucuronidation and sulfation, primarily in liver as well as other tissues and regulated by 
gut microbiota metabolism. Measurement of these biotransformed metabolites effects compared to DMY has yet to be reported and might help identify more potential analogues in the future.

DMY has been demonstrated to not only exert neuroprotection, anticancer, antimicrobial, detoxification, cell death-mediating, as well as lipid and glucose metabolism-regulatory activities but also low toxicity through in vivo or in vitro experiments, posing it as potential ingredient in supplements or food industries. However, more clinical studies are needed to assess it long term effect in humans especially if to be consumed in nutraceuticals over long period of time.

In cell and animal models, DMY biological actions have been sufficiently confirmed. However, a lack of clinical studies significantly limits its application in clinical practice. With the advances in metabolomics and other omics, better monitoring of DMY metabolism can be achieved. With the development of multi-omics and bioinformatics, the intestinal flora has been confirmed to be closely related to human digestion, absorption, metabolism, immunity and other functions. As a member of the polyphenol family, there are few reports on the relationship between DMY and intestinal flora, as well as how it modulates gut homeostasis. In addition, understanding the inter individual variability in metabolism will aid in the successful development of the latter into health product (s).

Therefore, enhancing poor bioavailability, figuring out the underlying mechanism of action and obtaining more clinical evidence about DMY toxicity and pharmacological effects appear as the major future research needed to maximize DMY applications in the future.

\section{REFERENCES}

[1] Liu RH. Dietary Bioactive Compounds and Their Health Implications. Journal of Food Science. 2013;78(s1):18-25. doi:10.1111/1750-3841.12101.

[2] Cisneros-Zevallos L. The power of plants: how fruit and vegetables work as source of nutraceuticals and supplements. International Journal of Food Sciences and Nutrition. 2021;72(5):660-664. doi:10.1080/09637486.2020.1852194.

[3] Li AN, Li S, Zhang YJ, Xu XR, Chen YM, Li HB. Resources and Biological Activities of Natural Polyphenols. Nutrients. 2014;6(12):6020-6047. doi:10.3390/nu6126020.

[4] Tomás-Barberán FA, Andrés-Lacueva C. Polyphenols and Health: Current State and Progress. Journal of Agricultural and Food Chemistry. 2012;60(36):8773-8775. doi:10.1021/jf300671j.

[5] Panche AN, Diwan AD, Chandra SR. Flavonoids: an overview. Journal of Nutritional Science. 2016;5:e47. doi:10.1017/jns.2016.41.

[6] Gao W, Lee SU, Li J, Lee JW. Development of Improved Process with Treatment of Cellulase for Isolation of Ampelopsin from Dried Fruits of Ampelopsis grossedentata. BioResources. 2016;11(1):2712-2722. doi:10.15376/biores.11.1.2712-2722.

[7] Sun CC, Li Y, Yin ZP, Zhang QF. Physicochemical properties of dihydromyricetin and the effects of ascorbic acid on its stability and bioavailability. Journal of the Science of Food and Agriculture. 2020;101(9):3862-3869.

[8] Maieves HA, López-Froilán R, Morales P, Pérez-Rodríguez ML, Ribani RH, Cámara M, et al. Antioxidant phytochemicals of Hovenia dulcis Thunb. peduncles in different maturity stages. Journal of Functional Foods. 2015;18:1117-1124. doi:10.1016/j.jff.2015.01.044.

[9] Mi JH, Nim HL, Jin HJ. Hovenia dulcis Thunb. and its active compound ampelopsin inhibit angiogenesis through suppression of VEGFR2 signaling and HIF- $1 \alpha$ expression. Oncol Rep. 2017;38(6):3430-3438.

[10] Sferrazza G, Brusotti G, Zonfrillo M, Temporini C, Tengattini S, Bononi M, et al. Hovenia dulcis Thumberg: Phytochemistry, Pharmacology, Toxicology and Regulatory Framework for Its Use in the European Union. Molecules. 2021;26(4):903-903. doi:10.3390/molecules26040903.

[11] Liang X, Wu YP, Qiu JH, Zhong K, Gao H. A Potent Antibrowning Agent from Pine Needles of Cedrus deodara: 2R,3R-Dihydromyricetin. Journal of Food Science. 2014;79(9):1643-1648. doi:10.1111/1750-3841.12583.

[12] Liu H, Zhao W, Hu Q, Zhao L, Wei Y, Pi C, et al. Gastric floating sustained-release tablet for dihydromyricetin: Development, characterization, and pharmacokinetics study. Saudi Pharmaceutical Journal. 2019;27(7):1000-1008. doi:10.1016/j.jsps.2019.08.002.

[13] Hu H, Luo F, Wang M, Fu Z, Shu X. New Method for Extracting and Purifying Dihydromyricetin from Ampelopsis grossedentata. ACS Omega. 2020;5(23):13955-13962. doi:10.1021/acsomega.0c01222.

[14] Zhang J, Chen Y, Luo H, Sun L, Xu M, Yu J, et al. Recent update on the pharmacological effects and mechanisms of dihydromyricetin. Frontiers in Pharmacology. 2018;9:1-11. doi:10.3389/fphar.2018.01204.

[15] Park JN, Kim JH. Kinetic and thermodynamic characteristics of fractional precipitation of $(+)$ dihydromyricetin. Process Biochemistry. 2017;53:224-231. doi:10.1016/j.procbio.2016.11.014.

[16] Wang C, Xiong W, Perumalla SR, Fang J, Sun CC. Solid-state characterization of optically pure (+)Dihydromyricetin extracted from Ampelopsis grossedentata leaves. International Journal of Pharmaceutics. 2016;511(1):245-252.

[17] Umair M, Jabbar S, Sultana T, Ayub Z, Abdelgader SA, Xiaoyu Z, et al. Chirality of the biomolecules enhanced its stereospecific action of dihydromyricetin enantiomers. Food Science and Nutrition. 2020;8(9):4843-4856.

[18] Tong Q, Hou X, Fang J, Wang W, Xiong W, Liu X, et al. Determination of dihydromyricetin in rat plasma by LC-MS/MS and its application to a pharmacokinetic study. Journal of Pharmaceutical and Biomedical Analysis. 2015;114:455-461. doi:10.1016/j.jpba.2015.06.030.

[19] Chen, Liang, Shi M, Lv C, Song Y, Wu Y, et al. Dihydromyricetin Acts as a Potential Redox Balance Mediator in Cancer Chemoprevention. Mediators of Inflammation. 2021;2021:1-18. doi:10.1155/2021/6692579.

[20] Zhang P, Cai S, Song L, Zhang L, Fan H, Zhou L, et al. Solubility of dihydromyricetin in ethanol and water mixtures from 288.15 to $323.15 K$. Journal of Molecular Liquids. 2015;211:197-202. doi:10.1016/j.molliq.2015.07.007.

[21] Chen Y, Luo HQ, Sun LL, Xu MT, Yu J, Liu LL, et al. Dihydromyricetin Attenuates Myocardial Hypertrophy Induced by Transverse Aortic Constriction via Oxidative Stress Inhibition and SIRT3 Pathway Enhancement. International Journal of Molecular Sciences. 2018;19(9). doi:10.3390/ijms19092592.

[22] Wu X, Li M, Xiao Z, Daglia M, Dragan S, Delmas D, et al. Dietary polyphenols for managing cancers: What have we ignored? Trends in Food Science and Technology. 2020;101:150-164.

[23] Xie K, He X, Chen K, Chen J, Sakao K, Hou DX. Antioxidant Properties of a Traditional Vine Tea. Ampelopsis grossedentata 
Antioxidants. 2019;8(8). doi:10.3390/antiox8080295.

[24] Zheng Q, Fan J. Effect of Dihydromyricetin on the Stability of Polypropylene in Natural Weathering Tests. Journal of Macromolecular Science, Part B. 2016;55(4):426-431. doi:10.1080/00222348.2016.1153404.

[25] Wang C, Tong Q, Hou X, Hu S, Fang J, Sun CC. Enhancing Bioavailability of Dihydromyricetin through Inhibiting Precipitation of Soluble Cocrystals by a Crystallization Inhibitor. Crystal Growth and Design. 2016;16(9):5030-5039.

[26] Xiang D, Wang CG, Wang WQ, Shi CY, Xiong W, Wang $\mathrm{MD}$, et al. Gastrointestinal stability of dihydromyricetin, myricetin, and myricitrin: an in vitro investigation. International Journal of Food Sciences and Nutrition. 2017;68(6):704-711. doi:10.1080/09637486.2016.1276518.

[27] Maini S, Hodgson HL, Krol ES. The UVA and Aqueous Stability of Flavonoids Is Dependent on B-Ring Substitution. Journal of Agricultural and Food Chemistry. 2012;60(28):6966-6976. doi:10.1021/jf3016128.

[28] Wang L, Qin Y, Wang Y, Zhou Y, Liu B. Interaction between iron and dihydromyricetin extracted from vine tea. Food Science \& Nutrition. 2020;8(11):5926-5933.

[29] Huang J, He Z, Cheng R, Cheng Z, Wang S, Wu X, et al. Assessment of binding interaction dihydromyricetin and myricetin with bovine lactoferrin and effects on antioxidant activity. Spectrochimica Acta - Part A: Molecular and Biomolecular Spectroscopy. 2020;243:118731-118731. doi:10.1016/j.saa.2020.118731.

[30] Chen T, Zhu S, Shang Y, Ge C, Jiang G. Binding of dihydromyricetin to human hemoglobin: Fluorescence and circular dichroism studies. Spectrochimica Acta Part A: Molecular and Biomolecular Spectroscopy. 2012;93:125-130. doi:10.1016/j.saa.2012.02.109.

[31] Yu X, Liu R, Yang F, Ji D, Li X, Chen J, et al. Study on the interaction between dihydromyricetin and bovine serum albumin by spectroscopic techniques. Journal of Molecular Structure. 2011;985(2-3):407-412. doi:10.1016/j.molstruc.2010.11.034.

[32] Xiao J. Stability of dietary polyphenols: It's never too late to mend? Food and Chemical Toxicology. 2018;119:3-5. doi:10.1016/j.fct.2018.03.051.

[33] Xiao JB, Högger P. Stability of dietary polyphenols under the cell culture conditions: Avoiding erroneous conclusions. Journal of Agricultural and Food Chemistry. 2015;63(5):15471557. doi:10.1021/jf505514d.

[34] Cao H, Högger P, Arroo R, Xiao J. Flavonols with a catechol or pyrogallol substitution pattern on ring $B$ readily form stable dimers in phosphate buffered saline at four degrees celsius. Food Chemistry. 2020;311:125902-125902.

[35] He GX, Pei G, Yang WL, Li B. Determination of dihydromyricetin in different parts of Ampelopsis grossedentata in different seasons by HPLC. Chinese Traditional Patent Medicine. 1992;(03):0-0.

[36] Yu QP, He LL, Wang JS, Zhang N, Kong Q, Yu ZW. Dynamics Changes of Functional Ingredients from Cultivated Ampelopsis grossedentata. Chinese Journal of Experimental Traditional Medical Formulae. 2014;p. 9-9.

[37] Ke Z, Zhu H, S, Zou Z, F D. Study on Extraction and Separation of Dihydromyricetin from Vine Tea [Ampelopsis grossedentata (Hand-Mazz) WT wang. Agricultural Biotechnology. 2020;9(1):2164-4993.

[38] Yao Y, Zhang M, He L, Wang Y, Chen S. Evaluation of General Synthesis Procedures for Bioflavonoid-Metal Complexes in AirSaturated Alkaline Solutions. Frontiers in Chemistry. 2020;8:1-
11.

[39] Muhammad U, Lu H, Wang J, Han J, Zhu X, Lu Z, et al. Optimizing the maximum recovery of dihydromyricetin from Chinese vine tea, ampelopsis grossedentata, using response surface methodology. Molecules. 2017;(12):22-22. doi:10.3390/molecules22122250.

[40] Yanni L, Xiaobin Z, Duowei L. Optimization of Dihydromyricetin ultrasonic extraction technology from Ampelopsis grossedentata leaves. Guizhou Agricultural Sciences. 2018;46(5):123-126.

[41] Li W, Chen Y, Xu X, Zheng C, Zhao J. Optimization of dynamic microwave-assisted extraction of dihydromyricetin from Ampelopsis grossedentata using response surface methodology. Journal of the Chemical Society of Pakistan. 2018;40(6):10281034.

[42] Wang L, Zhou Y, Wang Y, Qin Y, Liu B, Bai M. Two green approaches for extraction of dihydromyricetin from Chinese vine tea using $\beta$-Cyclodextrin-based and ionic liquid-based ultrasonic-assisted extraction methods. . Food and Bioproducts Processing. 2019;116:1-9. Available from: https://doi.org/10. 1016/j.fbp.2019.04.005. doi:10.1016/j.fbp.2019.04.005.

[43] Astray G, Gonzalez-Barreiro C, Mejuto JC, Rial-Otero R, Simal-Gándara J. A review on the use of cyclodextrins in foods. Food Hydrocolloids. 2009;23(7):1631-1640. doi:10.1016/j.foodhyd.2009.01.001.

[44] Liu B, Ma Y, Yuan C, Su C, Hu L, Wang J. Characterization, stability and antioxidant activity of the inclusion complex of dihydromyricetin with hydroxypropyl- $\beta$-cyclodextrin. Journal of Food Biochemistry. 2012;36(5):634-641. doi:10.1111/j.17454514.2011.00577.x.

[45] Wang J, Wang K, Huang C, Lin D, Zhou Y, Wu Y, et al. SIRT3 Activation by Dihydromyricetin Suppresses Chondrocytes Degeneration via Maintaining Mitochondrial Homeostasis. International Journal of Biological Sciences. 2018;14(13):18731882. doi:10.7150/ijbs.27746.

[46] Samsonowicz M, Regulska E, Kalinowska M. Hydroxyflavone metal complexes - molecular structure, antioxidant activity and biological effects. Chemico-Biological Interactions. 2017;273:245-256. doi:10.1016/j.cbi.2017.06.016.

[47] Wu C, Zheng XP, Chen LL. Study on Antioxidant Activity of Dihydromyricetin-Zinc(II) Complex. Advanced Materials Research. 2011;183:863-867. doi:10.4028/www.scientific.net/AMR.183-185.863.

[48] Du Q, Cai W, Xia M, Ito Y. Purification of (+)-dihydromyricetin from leaves extract of Ampelopsis grossedentata using highspeed countercurrent chromatograph with scale-up triple columns. Journal of Chromatography A. 2002;973(1-2):217220. doi:10.1016/S0021-9673(02)01092-0.

[49] Ameen F, Alyahya SA, Bakhrebah MA, Nassar MS, Aljuraifani A. Flavonoid dihydromyricetin-mediated silver nanoparticles as potential nanomedicine for biomedical treatment of infections caused by opportunistic fungal pathogens. Research on Chemical Intermediates. 2018;44(9):5063-5073. doi:10.1007/s11164-0183409-X.

[50] Lin Y, Fan J, Ruan L, Bi J, Yan Y, Wang T, et al. Semi-preparative separation of dihydromyricetin enantiomers by supercritical fluid chromatography and determination of anti-inflammatory activities. Journal of Chromatography A. 1606;p. 460386460386. doi:10.1016/j.chroma.2019.460386.

[51] Li X, Cao M, Ma W, Jia C, Li J, Zhang M, et al. Annotation of genes involved in high level of dihydromyricetin production in vine tea (Ampelopsis grossedentata) by transcriptome analysis. 
BMC Plant Biology. 2020;20(1):1-12.

[52] Milke L, Aschenbrenner J, Marienhagen J, Kallscheuer N. Production of plant-derived polyphenols in microorganisms: current state and perspectives. Applied Microbiology and Biotechnology. 2018;102(4):1575-1585. doi:10.1007/s00253018-8747-5.

[53] Li H, Li Q, Liu Z, Yang K, Chen Z, Cheng Q, et al. The Versatile Effects of Dihydromyricetin in Health. 2017; doi:10.1155/2017/1053617.

[54] Li G, Li H, Lyu Y, Zeng W, Zhou J. Enhanced Biosynthesis of Dihydromyricetin in Saccharomyces cerevisiae by Coexpression of Multiple Hydroxylases. Journal of Agricultural and Food Chemistry. 2020;68(48):14221-14229.

[55] Xiang D, Fan L, Hou X, Long, Xiong W, Shi C, et al. Uptake and Transport Mechanism of Dihydromyricetin Across Human Intestinal Caco-2 Cells. Journal of Food Science. 2018;83(7):1941-1947. doi:10.1111/1750-3841.14112.

[56] Barthe L, Woodley J, Houin G. Gastrointestinal absorption of drugs: methods and studies. Fundamental \& Clinical Pharmacology. 1999;13(2):154-168. doi:10.1111/j.14728206.1999.tb00334.x.

[57] Li M, Dyett B, Zhang X. Automated Femtoliter Droplet-Based Determination of Oil-Water Partition Coefficient. Analytical Chemistry. 2019;(16):10371-10375. doi:10.1021/acs.analchem.9b02586.

[58] Yu Z, Chen Z, Li Q, Yang K, Huang Z, Wang W, et al. What dominates the changeable pharmacokinetics of natural sesquiterpene lactones and diterpene lactones: a review focusing on absorption and metabolism. Drug Metabolism Reviews. 2020;0(0):1-19.

[59] Hidalgo IJ, Raub TJ, Borchardt RT. Characterization of the Human Colon Carcinoma Cell Line (Caco-2) as a Model System for Intestinal Epithelial Permeability. Gastroenterology. 1989;96(2):80072-80073. doi:10.1016/S0016-5085(89)80072-1.

[60] Liu L, Zhou M, Lang H, Zhou Y, Mi M. Dihydromyricetin enhances glucose uptake by inhibition of MEK/ERK pathway and consequent down-regulation of phosphorylation of PPAR $\gamma$ in 3T3-L1 cells. Journal of Cellular and Molecular Medicine. 2018;22(2):1247-1256. doi:10.1111/jcmm.13403.

[61] Schinkel AH, Jonker JW. Mammalian drug efflux transporters of the ATP binding cassette (ABC) family: an overview. Advanced Drug Delivery Reviews. 2012;64:138-153. doi:10.1016/j.addr.2012.09.027.

[62] Naeem A, Ming Y, Pengyi H, Jie KY, Yali L, Haiyan Z, et al. The fate of flavonoids after oral administration: a comprehensive overview of its bioavailability. Critical Reviews in Food Science and Nutrition. 2021;0(0):1-18. doi:10.1080/10408398.2021.1898333.

[63] Huang Y, Zhao J, Jian W, Wang G. Effects of verapamil on the pharmacokinetics of dihydromyricetin in rats and its potential mechanism. Xenobiotica. 2018;48(8):839-844. doi:10.1080/00498254.2017.1366576.

[64] Zhang Y, Que S, Yang X, Wang B, Qiao L, Zhao Y. Isolation and identification of metabolites from dihydromyricetin. Magnetic Resonance in Chemistry. 2007;45(11):909-916. doi:10.1002/mrc.2051.

[65] Liu L, Yin X, Wang X, Li X. Determination of dihydromyricetin in rat plasma by LC-MS/MS and its application to a pharmacokinetic study. Pharmaceutical Biology. 2017;55(1):657-662. doi:10.1080/13880209.2016.1266669.

[66] Fan L, Tong Q, Dong W, Yang G, Hou X, Xiong W, et al. Tissue Distribution, Excretion, and Metabolic Profile of Dihydromyricetin, a Flavonoid from Vine Tea (Ampelopsis grossedentata) after Oral Administration in Rats. Journal of Agricultural and Food Chemistry. 2017;65(23):4597-4604. doi:10.1021/acs.jafc.7b01155.

[67] Catalkaya G, Venema K, Lucini L, Rocchetti G, Delmas D, Daglia $\mathrm{M}$, et al. Interaction of dietary polyphenols and gut microbiota: Microbial metabolism of polyphenols, influence on the gut microbiota, and implications on host health. Food Frontiers. 2020;1(2):109-133.

[68] Fan L, Zhao X, Tong Q, Zhou X, Chen J, Xiong W, et al. Interactions of Dihydromyricetin, a Flavonoid from Vine Tea (Ampelopsis grossedentata) with Gut Microbiota. Journal of Food Science. 2018;83(5):1444-1453. doi:10.1111/17503841.14128.

[69] Liu H, Gan C, Shi H, Qu K, Jing L, Lu M, et al. Gastric floating pill enhances the bioavailability and drug efficacy of dihydromyricetin in vivo. Journal of Drug Delivery Science and Technology. 2021;61:102279-102279. doi:10.1016/j.jddst.2020.102279.

[70] Chen T, Zhu S, Lu Y, Cao H, Zhao Y, Jiang G, et al. Probing the Interaction of Anti-Cancer Agent Dihydromyricetin with Human Serum Albumin: A typical Method Study. In AntiCancer Agents in Medicinal Chemistry. 2012;12(8):919-928.

[71] Ye J, Bao S, Zhao S, Zhu Y, Ren Q, Li R, et al. Self-Assembled Micelles Improve the Oral Bioavailability of Dihydromyricetin and Anti-Acute Alcoholism Activity. AAPS PharmSciTech. 2021;22(3):111-111. doi:10.1208/s12249-021-01983-2.

[72] Sun CC, Su H, Zheng GD, Wang WJ, Yuan E, Zhang QF. Fabrication and characterization of dihydromyricetin encapsulated zein-caseinate nanoparticles and its bioavailability in rat. Food Chemistry. 2020;330:127245-127245.

[73] Cao SL, Deng X, Xu P, Huang ZX, Zhou J, Li XH, et al. Highly Efficient Enzymatic Acylation of Dihydromyricetin by the Immobilized Lipase with Deep Eutectic Solvents as Cosolvent. Journal of Agricultural and Food Chemistry. 2017;65(10):20842088. doi:10.1021/acs.jafc.7b00011.

[74] Phang-Lyn S, Llerena VA. 2020.

[75] Li, Houkai, He J, Jia W. The influence of gut microbiota on drug metabolism and toxicity. Expert Opinion on Drug Metabolism \& Toxicology. 2016;12(1):31-40. doi:10.1517/17425255.2016.1121234.

[76] Meyer UA. Overview of enzymes of drug metabolism. Journal of Pharmacokinetics and Biopharmaceutics. 1996;24(5):449-459. doi:10.1007/BF02353473.

[77] Ge H, Guan S, Shen Y, Sun M, Hao Y, He L, et al. Dihydromyricetin affects BDNF levels in the nervous system in rats with comorbid diabetic neuropathic pain and depression. Scientific Reports. 2019;9(1):14619-14619. doi:10.1038/s41598019-51124-w.

[78] Guan S, Shen Y, Ge H, Xiong W, He L, Liu L, et al. Dihydromyricetin Alleviates Diabetic Neuropathic Pain and Depression Comorbidity Symptoms by Inhibiting P2X7 Receptor. Frontiers in Psychiatry. 2019;10. doi:10.3389/fpsyt.2019.00770.

[79] Sun P, Yin JB, Liu LH, Guo J, Wang SH, Qu CH, et al. Protective role of Dihydromyricetin in Alzheimer's disease rat model associated with activating AMPK/SIRT1 signaling pathway. Bioscience Reports. 2019;(1):39-39. doi:10.1042/BSR20180902.

[80] Liu M, Guo H, Li Z, Zhang C, Zhang X, Cui Q, et al. Molecular Level Insight Into the Benefit of Myricetin and Dihydromyricetin Uptake in Patients With Alzheimer's Diseases. In Frontiers in Aging Neuroscience. 2020;12:601303-601303. 
[81] Jia L, Wang Y, Sang J, Cui W, Zhao W, Wei W, et al. Dihydromyricetin Inhibits $\alpha$-Synuclein Aggregation, Disrupts Preformed Fibrils, and Protects Neuronal Cells in Culture against Amyloid-Induced Cytotoxicity. Journal of Agricultural and Food Chemistry. 2019;67(14):3946-3955. doi:10.1021/acs.jafc.9b00922.

[82] Qian J, Wang X, Cao J, Zhang W, Lu C, Chen X. Dihydromyricetin attenuates D-galactose-induced brain aging of mice via inhibiting oxidative stress and neuroinflammation. Neuroscience Letters. 2021;756:135963-135963. doi:10.1016/j.neulet.2021.135963.

[83] Liu CM, Yang W, Ma JQ, Yang HX, Feng ZJ, Sun JM, et al. Dihydromyricetin Inhibits Lead-Induced Cognitive Impairments and Inflammation by the Adenosine $5^{\prime}$ Monophosphate-Activated Protein Kinase Pathway in Mice. Journal of Agricultural and Food Chemistry. 2018;66(30):79757982.

[84] Fan KJ, Yang B, Liu Y, Tian XD, Wang B. Inhibition of human lung cancer proliferation through targeting stromal fibroblasts by dihydromyricetin. Mol Med Rep. 2017;16(6):9758-9762. doi:10.3892/mmr.2017.7802.

[85] Zuo Y, Lu Y, Xu Q, Sun D, Liang X, Li X, et al. Inhibitory effect of dihydromyricetin on the proliferation of JAR cells and its mechanism of action. Oncol Lett. 2020;20(1):357-363. doi:10.3892/ol.2020.11546.

[86] Liang J, Wu J, Wang F, Zhang P, Zhang X. Semaphoring $4 \mathrm{D}$ is required for the induction of antioxidant stress and anti-inflammatory effects of dihydromyricetin in colon cancer. International Immunopharmacology. 2019;67:220230. doi:10.1016/j.intimp.2018.12.025.

[87] Chen L, Yang, Zhou ZS, Deng YZ, Jiang Y, Tan P, et al. Dihydromyricetin inhibits cell proliferation, migration, invasion and promotes apoptosis via regulating miR-21 in Human Cholangiocarcinoma Cells. Journal of Cancer. 2020;11(19):5689-5699.

[88] Jiang L, Ye WC, Li Z, Yang Y, Dai W, Li M. Anticancer effects of dihydromyricetin on the proliferation, migration, apoptosis and in vivo tumorigenicity of human hepatocellular carcinoma Hep3B cells. BMC Complementary Medicine and Therapies. 2021;21(1):194-194. doi:10.1186/s12906-021-03356-5.

[89] Zhang Z, Zhang H, Chen S, Xu Y, Yao A, Liao Q, et al. Dihydromyricetin induces mitochondria-mediated apoptosis in HepG2 cells through down-regulation of the Akt/Bad pathway. Nutrition Research. 2017;38:27-33. doi:10.1016/j.nutres.2017.01.003.

[90] Tan M, Jiang B, Wang H, Ouyang W, Chen X, Wang $\mathrm{T}$, et al. Dihydromyricetin induced lncRNA MALAT1TFEB-dependent autophagic cell death in cutaneous squamous cell carcinoma. Journal of Cancer. 2019;10(18):4245-4255. doi:10.7150/jca.32807.

[91] Wei L, Sun X, Qi X, Zhang Y, Li Y, Xu Y. Dihydromyricetin Ameliorates Cardiac Ischemia/Reperfusion Injury through Sirt3 Activation. BioMed Research International. 2019;6803943. doi:10.1155/2019/6803943.

[92] Song Q, Liu L, Yu J, Zhang J, Xu M, Sun L, et al. Dihydromyricetin attenuated Ang II induced cardiac fibroblasts proliferation related to inhibitory of oxidative stress. European Journal of Pharmacology. 2017;807:159-167. doi:10.1016/j.ejphar.2017.04.014.

[93] Luo Y, Lu S, Dong X, Xu L, Sun G, Sun X. Dihydromyricetin protects human umbilical vein endothelial cells from injury through ERK and Akt mediated Nrf2/HO-1 signaling pathway. Apoptosis. 2017;22(8):1013-1024. doi:10.1007/s10495-0171381-3.
[94] Zhang X, Wang L, Peng L, Tian X, Qiu X, Cao H, et al. Dihydromyricetin protects HUVECs of oxidative damage induced by sodium nitroprusside through activating PI3K/Akt/FoxO3a signalling pathway. Journal of Cellular and Molecular Medicine. 2019;23(7):4829-4838. doi:10.1111/jcmm.14406.

[95] Li Q, Wang J, Zhu X, Zeng Z, Wu X, Xu Y, et al. Dihydromyricetin prevents monocrotaline-induced pulmonary arterial hypertension in rats. Biomedicine \& Pharmacotherapy. 2017;96:825-833. doi:10.1016/j.biopha.2017.10.007.

[96] Sun Z, Lu W, Lin N, Lin H, Zhang J, Ni T, et al. Dihydromyricetin alleviates doxorubicin-induced cardiotoxicity by inhibiting NLRP3 inflammasome through activation of SIRT1. Biochemical Pharmacology. 2020;175:113888. doi:10.1016/j.bcp.2020.113888.

[97] Dong S, Ji J, Hu L, Wang H. Dihydromyricetin alleviates acetaminophen-induced liver injury via the regulation of transformation, lipid homeostasis, cell death and regeneration. Life Sciences. 2019;227:20-29. doi:10.1016/j.1fs.2019.04.019.

[98] Yan Y, Wang K, Tang X, Gao J, Wen B. Phytochemicals protect L02 cells against hepatotoxicity induced by emodin via the Nrf2 signaling pathway. Toxicology Research. 2019;8(6):1028-1034. doi:10.1039/c9tx00220k.

[99] Carry E, Kshatriya D, Silva J, Davies DL, Yuan B, Wu Q, et al. Identification of Dihydromyricetin and Metabolites in Serum and Brain Associated with Acute Anti-Ethanol Intoxicating Effects in Mice. International Journal of Molecular Sciences. 2021;(14):22-22. doi:10.3390/ijms22147460.

[100] Chang Y, Yuan L, Liu J, Muhammad I, Cao C, Shi C, et al. Dihydromyricetin attenuates Escherichia coli lipopolysaccharide-induced ileum injury in chickens by inhibiting NLRP3 inflammasome and TLR4/NF- $\kappa \mathrm{B}$ signalling pathway. Veterinary Research. 2020;51(1). doi:10.1186/s13567020-00796-8.

[101] Long H, Xin Z, Zhang F, Zhai Z, Ni X, Chen J, et al. The cytoprotective effects of dihydromyricetin and associated metabolic pathway changes on deoxynivalenol treated IPEC-J2 cells. Food Chemistry. 2021;338. doi:10.1016/j.foodchem.2020.128116.

[102] on Nutrition EP, Turck D, Castenmiller J, Henauw SD, HirschErnst KI, Kearney J, et al. Safety of hot water extract of fruits and peduncles of Hovenia dulcis as a novel food pursuant to Regulation 1(EU) 2015/2283. EFSA Journal. 2020;18(8):e06196. doi:10.2903/j.efsa.2020.6196.

[103] Ren Z, Yan P, Zhu L, Yang H, Zhao Y, Kirby BP, et al. Dihydromyricetin exerts a rapid antidepressant-like effect in association with enhancement of BDNF expression and inhibition of neuroinflammation. Psychopharmacology. 2018;235(1):233-244. doi:10.1007/s00213-017-4761-z.

[104] Guo C, Cao T, Zheng L, Waddington JL, Zhen X. Development and characterization of an inducible Dicer conditional knockout mouse model of Parkinson's disease: validation of the antiparkinsonian effects of a sigma-1 receptor agonist and dihydromyricetin. Acta Pharmacologica Sinica. 2020;41(4):499507.

[105] Kalluri R. The biology and function of fibroblasts in cancer. Nature Reviews Cancer. 2016;16(9):582-598. doi:10.1038/nrc.2016.73.

[106] Davidson S, Coles M, Thomas T, Kollias G, Ludewig B, Turley $\mathrm{S}$, et al. Fibroblasts as immune regulators in infection, inflammation and cancer. Nature Reviews Immunology. 2021;p. 1-14. doi:10.1038/s41577-021-00540-Z. 
[107] Zhu XH, Lang HD, Wang XL, Hui SC, Zhou M, Kang C, et al. Synergy between dihydromyricetin intervention and irinotecan chemotherapy delays the progression of colon cancer in mouse models. Food \& Function. 2019;10(4):2040-2049. doi:10.1039/C8FO01756E.

[108] Sun Y, Wang C, Meng Q, Liu Z, Huo X, Sun P, et al. Targeting P-glycoprotein and SORCIN: Dihydromyricetin strengthens anti-proliferative efficiency of adriamycin via MAPK/ERK and $\mathrm{Ca} 2+-$ mediated apoptosis pathways in MCF-7/ADR and K562/ADR. Journal of Cellular Physiology. 2018;233(4):30663079. doi:10.1002/jcp.26087.

[109] Xu B, Huang S, Wang C, Zhang H, Fang S, Zhang Y. Anti-inflammatory effects of dihydromyricetin in a mouse model of asthma. Mol Med Rep. 2017;15(6):3674-3680. doi:10.3892/mmr.2017.6428.

[110] Gao J, Shi N, Guo H, Gao J, Tang X, Yuan S, et al. UPLC-Q-TOF/MS-Based Metabolomics Approach to Reveal the Hepatotoxicity of Emodin and Detoxification of Dihydromyricetin. ACS Omega. 2021;6(8):5348-5358. doi:10.1021/acsomega.0c05488.

[111] Shen Y, Lindemeyer AK, Gonzalez C, Shao XM, Spigelman I, Olsen RW, et al. Dihydromyricetin As a Novel AntiAlcohol Intoxication Medication. The Journal of Neuroscience. 2012;32(1):390-401. doi:10.1523/JNEUROSCI.4639-11.2012.

[112] Han H, Dong Y, Ma X. Dihydromyricetin Protects Against Gentamicin-Induced Ototoxicity via PGC- $1 \alpha /$ SIRT3 Signaling in vitro. Frontiers in Cell and Developmental Biology. 2020;8:702-702.

[113] Chu J, Wang X, Bi H, Li L, Ren M, Wang J. Dihydromyricetin relieves rheumatoid arthritis symptoms and suppresses expression of pro-inflammatory cytokines via the activation of Nrf2 pathway in rheumatoid arthritis model. International Immunopharmacology. 2018;59:174-180. doi:10.1016/j.intimp.2018.04.001.

[114] Jia R, Ma J, Meng W, Wang N. Dihydromyricetin inhibits caerulin-induced TRAF3-p38 signaling activation and acute pancreatitis response. Biochemical and Biophysical Research Communications. 2018;503(3):1696-1702. doi:10.1016/j.bbrc.2018.07.101.

[115] Wang HL, Xing GD, Qian Y, Sun XF, Zhong JF, Chen KL. Dihydromyricetin attenuates heat stress-induced apoptosis in dairy cow mammary epithelial cells through suppressing mitochondrial dysfunction. Ecotoxicology and Environmental Safety. 2021;214. doi:10.1016/j.ecoenv.2021.112078.

[116] Zhou DZ, Sun HY, Yue JQ, Peng Y, Chen YM, Zhong ZJ. Dihydromyricetin induces apoptosis and cytoprotective autophagy through ROS-NF- $\kappa \mathrm{B}$ signalling in human melanoma cells. Free Radical Research. 2017;51(5):517-528. doi:10.1080/10715762.2017.1328552.

[117] Ye X, Pang Z, Zhu N. Dihydromyricetin attenuates hypertrophic scar formation by targeting activin receptor-like kinase 5. European Journal of Pharmacology. 2019;852:58-67. doi:10.1016/j.ejphar.2019.02.039.

[118] Tian Y, Sang H, Liu M, Chen F, Huang Y, Li L, et al. Dihydromyricetin is a new inhibitor of influenza polymerase PB2 subunit and influenza-induced inflammation. Microbes and Infection. 2020;22(6):254-262.

[119] Huang W, Xie J. Antibacterial Effect of Dihydromyricetin on Specific Spoilage Organisms of Hybrid Grouper. Journal of Food Quality. 2021;2021:5569298. doi:10.1155/2021/5569298.

[120] Wu YP, Bai JR, Grosu E, Zhong K, Liu LJ, Tang MM, et al. Inhibitory Effect of 2R,3R-Dihydromyricetin on Biofilm
Formation by Staphylococcus aureus. Foodborne Pathogens and Disease. 2018;15(8):475-480. doi:10.1089/fpd.2017.2405.

[121] Sun B, Tan D, Pan D, Baker MR, Liang Z, Wang Z, et al. Dihydromyricetin Imbues Antiadipogenic Effects on 3T3-L1 Cells via Direct Interactions with $78-\mathrm{kDa}$ Glucose-Regulated Protein. The Journal of Nutrition. 2021;151(7):1717-1725. doi:10.1093/jn/nxab057.

[122] Yao M, Teng H, Lv Q, Gao H, Guo T, Lin Y, et al. Antihyperglycemic effects of dihydromyricetin in streptozotocininduced diabetic rats. Food Science and Human Wellness. 2021;10:155-162. doi:10.1016/j.fshw.2021.02.004.

[123] Chen, Shihui, Zhao X, Wan J, Ran L, Qin Y, et al. Dihydromyricetin improves glucose and lipid metabolism and exerts anti-inflammatory effects in nonalcoholic fatty liver disease: A randomized controlled trial. Pharmacological Research. 2015;99:74-81. doi:10.1016/j.phrs.2015.05.009.

[124] Fan X, Zeng Y, Fan Z, Cui L, Song W, Wu Q, et al. Dihydromyricetin promotes longevity and activates the transcription factors FOXO and AOP in Drosophila. Aging. 2020;13(1):460-476. doi:10.18632/aging.202156.

[125] Zhou Y, Hu Y, Zang B, Qiu F, Liu X, Nie Y, et al. Toxicological assessment on Ampelopsis grossedentata and its immune regulation study. Practical Preventive Medicine. 2001;8(6):412414.

[126] Su DL, Huang JH, Yao MJ. The acute toxicological evaluation of dihydromyricetin and its control effect for alcoholic hepatic injury. Hun Agricultural Sci. 2009;p. 90-93.

[127] Zhong Z, Zhou G, Chen X. The rat chronic toxicity test of total flavone of Ampelopsis grossedentata from Guangxi. Lishizhen Medicine \& Materia Medical Research. 2003;14(4):193-195.

[128] Silva FAP, Estévez M, Ferreira VCS, Silva SA, Lemos LTM, Ida EI, et al. Protein and lipid oxidations in jerky chicken and consequences on sensory quality. LWT. 2018;97:341-348. doi:10.1016/j.lwt.2018.07.022.

[129] Zhang X, Xu Y, Xue H, Jiang GC, Liu XJ. Antioxidant activity of vine tea (Ampelopsis grossedentata) extract on lipid and protein oxidation in cooked mixed pork patties during refrigerated storage. Food Science \& Nutrition. 2019;7(5):17351745. doi:10.1002/fsn3.1013.

[130] Jia C, Zhang M, Ma W, Li J, Zhao S, Xiong S, et al. Evaluation of antioxidant properties of the different tissues of vine tea (Ampelopsis grossedentata) in stripped canola oil and sunflower oil. Journal of Food Science. 2020;85(4):1082-1089.

[131] Moon KM, Kwon EB, Lee B, Kim CY. Recent trends in controlling the enzymatic browning of fruit and vegetable products. Molecules. 2020;25(12):2754-2754. doi:10.3390/molecules25122754.

[132] Tinello F, Lante A. Recent advances in controlling polyphenol oxidase activity of fruit and vegetable products. Innovative Food Science \& Emerging Technologies. 2018;50:73-83. doi:10.1016/j.ifset.2018.10.008.

[133] Díaz-Montes E, Castro-Muñoz R. Edible films and coatings as food-quality preservers: An overview. Foods. 2021;10(2):249249. doi:10.3390/foods 10020249 .

[134] Rodríguez GM, Sibaja JC, Espitia PJP, Otoni CG. Antioxidant active packaging based on papaya edible films incorporated with Moringa oleifera and ascorbic acid for food preservation. Food Hydrocolloids. 2020;103:105630-105630.

[135] Xie W, Du Y, Yuan S, Pang J. Dihydromyricetin incorporated active films based on konjac glucomannan and gellan gum. International Journal of Biological Macromolecules. 2021;180:385-391. doi:10.1016/j.ijbiomac.2021.02.185. 
[136] Xu J, Li X, Xu Y, Wang A, Xu Z, Wu X, et al. DihydromyricetinLoaded Pickering Emulsions Stabilized by Dialdehyde Cellulose Nanocrystals for Preparation of Antioxidant Gelatin-Based Edible Films. Food and Bioprocess Technology. 2021;Available from: https://doi.org/10.1007/s11947-021-02664-5. doi:10.1007/s11947-021-02664-5.

[137] Koszucka A, Nowak A. Thermal processing foodrelated toxicants: a review. Critical Reviews in Food Science and Nutrition. 2019;59(22):3579-3596. doi:10.1080/10408398.2018.1500440.

[138] Zhou B, Zhao Y, Wang X, Fan D, Cheng K, Wang M. Unraveling the inhibitory effect of dihydromyricetin on heterocyclic aromatic amines formation. Journal of the Science of Food and Agriculture. 2018;98(5):1988-1994. doi:10.1002/jsfa.8682.

[139] Teng J, Liu X, Hu X, Zhao Y, Tao NP, Wang M. Dihydromyricetin as a Functional Additive to Enhance Antioxidant Capacity and Inhibit the Formation of Thermally Induced Food Toxicants in a Cookie Model. Molecules. 2018;23(9):23. doi:10.3390/molecules23092184.

[140] Ma Q, Cai S, Jia Y, Sun X, Yi J, Du J. Effects of HotWater Extract from Vine Tea (Ampelopsis grossedentata) on Acrylamide Formation. Quality and Consumer Acceptability of Bread Foods. 2020;9(3):373-373.

[141] Geng S, Liu X, Ma H, Liu B, Liang G. Multi-scale stabilization mechanism of pickering emulsion gels based on dihydromyricetin/high-amylose corn starch composite particles. Food Chemistry. 2021;355:129660-129660. doi:10.1016/j.foodchem.2021.129660.

[142] Sarkar A, Dickinson E. Sustainable food-grade Pickering emulsions stabilized by plant-based particles. Current Opinion in Colloid \& Interface Science. 2020;49:69-81. 